2 New Covalent Organic Square Lattice Based on Porphyrin and 3 Tetraphenyl Ethylene Building Blocks toward High-Performance 4 Supercapacitive Energy Storage

5 Bidhan Chandra Patra ${ }^{\dagger, \#}$ and Santanu Bhattacharya ${ }^{*, \ddagger}, \dagger, \#$

${ }^{\ddagger}$ Department of Organic Chemistry, Indian Institute of Science, Bangalore 560012, India.

${ }^{\dagger}$ School of Applied and Interdisciplinary Sciences and ${ }^{\#}$ Technical Research Center, Indian Association for the Cultivation of Science, Jadavpur, Kolkata 700032, India.

\begin{tabular}{|c|c|c|}
\hline Section & & Page no \\
\hline S1 & Instrumentation & 2 \\
\hline S2 & Synthesis & 3 \\
\hline S3 & FT-IR spectroscopy & 15 \\
\hline S4 & Elemental Analysis & 15 \\
\hline S5 & BET plot & 16 \\
\hline S6 & Scanning Electron Microscopy (SEM) analysis & 17 \\
\hline S7 & Thermal stability of the PT-COF & 18 \\
\hline S8 & UV Vis and Photoluminescence study & 19 \\
\hline S9 & Conductivity measurement & 20 \\
\hline S10 & Electrochemical Study & $21-31$ \\
\hline S11 & Density of state (DOS) calculation & 31 \\
\hline S12 & Post cycle characterization of the PT-COF & 32 \\
\hline S13 & References & 34 \\
\hline Table S1 & Theoretical Calculations for P-XRD analysis of the PT-COF & $7-14$ \\
\hline Table S2 & $\begin{array}{l}\text { Experimental specific capacitance of the precursors (TFBE, } \\
\text { TAPP) and their physical mixtures. }\end{array}$ & 22 \\
\hline Table S3 & $\begin{array}{l}\text { Comparison table of specific capacitance of the PT-COF with } \\
\text { other literature reported COF. }\end{array}$ & 23 \\
\hline Table S4 & $\begin{array}{l}\text { Specific capacitance values of the PT-COF at three repeat } \\
\text { measurements obtained by CV analysis. }\end{array}$ & 24 \\
\hline Table S5 & $\begin{array}{l}\text { Specific capacitance values for three repeat measurement of the } \\
\text { PT-COF obtained by GCD analysis. }\end{array}$ & 25 \\
\hline Table S6 & $\begin{array}{l}\text { Specific capacitance values of PT-COF at } \mathrm{H}_{2} \mathrm{SO}_{4} \text { and } \mathrm{Na}_{2} \mathrm{SO}_{4} \text { at } \\
\text { different scan rates. }\end{array}$ & 26 \\
\hline
\end{tabular}


$12 \quad{ }^{1} \mathrm{H}$ and ${ }^{13} \mathrm{C}$ NMR spectra (liquid state) were recorded using Bruker $-400 / 500 \mathrm{MHz} \mathrm{NMR}$ and $100 \mathrm{MHz}$ spectrometer respectively. The ${ }^{13} \mathrm{C}$ solid state cross polarization magic angle spinning (CP-MAS) NMR spectrum was obtained using $100 \mathrm{MHz}$ Bruker Avance II spectrometer at a mass frequency of $8 \mathrm{kHz}$. X-Ray diffraction patterns of the powder samples were recorded Rigaku smart lab diffractometer using $\mathrm{Cu}-\mathrm{K} \alpha(0.15406 \mathrm{~nm})$ radiation at $40 \mathrm{kV}$ with $110 \mathrm{~mA}$ in $\mathrm{OD}$ detector. Volumetric Nitrogen adsorption/desorption experiments and micropore analysis was done at $77 \mathrm{~K}$ using Autosorb 1 (quantachrome, USA). Prior to surface area measurement, the sample was degassed under vacuum at $100{ }^{\circ} \mathrm{C}$ for $5 \mathrm{~h}$. Poresize distribution was obtained from the adsorption/desorption isotherm by using the carbon/slit-cylindrical pore model. A Gemini Zeiss 500 field-emission scanning electron microscope was used for visualizing the morphology of the particles. TEM images were taken on a UHR-FEG-TEM, JEOL; JEM $2100 \mathrm{~F}$ model of $200 \mathrm{kV}$ electron source. The sample was prepared by dropping a colloidal solution onto the carbon-coated copper grids followed by drying under high vacuum. Thermogravimetric (TGA) analysis of the samples has been done by TGA Instruments thermal analyser TA-SDT Q-600.

Electrochemical study:

Electrochemical measurement was carried out in a standard three electrode system with a 0.5 $\mathrm{M} \mathrm{H}_{2} \mathrm{SO}_{4}$ aqueous solution at $25{ }^{\circ} \mathrm{C}$ in a $\mathrm{CHI} 760 \mathrm{E}$ electrochemical work station $(\mathrm{CH}$ instrument, USA). Active material $(20 \mu \mathrm{g})$ coated glassy carbon electrode, Pt wire and a $\mathrm{AgCl} / \mathrm{Ag}(3 \mathrm{M} \mathrm{KCl})$ were considered as working, counter and reference electrodes respectively. The active material slurry was prepared by dispersing appropriate amount of a sample in isopropanol followed by addition of $5 \mathrm{wt}-\%$ Nafion solution. The geometric surface area of glassy carbon electrode used was $0.07 \mathrm{~cm}^{2}$ and active materials loading measured was 
$0.00285 \mathrm{mg} \mathrm{mm}^{-2}$. The cyclic voltammograms (CVs) were recorded within a potential window of $0 \mathrm{~V}$ to $1.0 \mathrm{~V}$ in the scan rate range of 2 to $100 \mathrm{mV} \mathrm{s}^{-1}$. Galvanostatic charge discharge (GCD) activity of electrode material was studied with different constant current density in a potential window of $0 \mathrm{~V}$ to $1.0 \mathrm{~V}$. The electrochemical impedance spectra (EIS) were recorded by applying a sinusoidal perturbation of $5 \mathrm{mV}$ in a frequency domain 1 to 100 $\mathrm{kHz}$.

Energy density and power density was calculated by the following equation

Energy density $=(1 / 2) \times \mathrm{C}_{s p} \times \mathrm{V}^{2}$

Power density $=\frac{\text { Energy density }}{\Delta \mathrm{T}}$

$\left(\mathrm{C}_{s p}=\right.$ specific capacitance, $\mathrm{V}=$ potential window, $\Delta \mathrm{t}=$ discharge time $)$

\section{S2. Synthesis:}<smiles>c1ccc(C(=C(c2ccccc2)c2ccccc2)c2ccccc2)cc1</smiles>

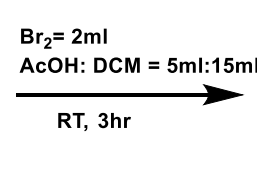

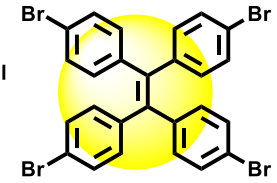

(2)

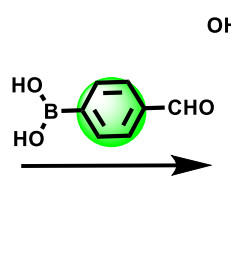

(3)

\section{Synthesis of tetrakis (4-bromophenyl) ethylene (2).}

Bromine (2.0 mL, $40.0 \mathrm{mmol}$ ) was added dropwise to a solution of 1, 1, 2, 2tetraphenylethylene $(1.66 \mathrm{~g}, 5.0 \mathrm{mmol})$ in glacial acetic acid $(5 \mathrm{~mL})$ and dichloromethane $(15$ $\mathrm{mL}$ ) at $0{ }^{\circ} \mathrm{C}$. Then the resulting mixture was stirred at room temperature for $3 \mathrm{~h}$, and then poured into $100 \mathrm{~mL}$ ice water. The organic part was extracted with $\mathrm{CH}_{2} \mathrm{Cl}_{2}$ and dried over anhydrous $\mathrm{Na}_{2} \mathrm{SO}_{4}$. The solvent was then removed under reduced pressure. The crude product was purified by recrystallization from methanol which furnished a white solid $(6.15 \mathrm{~g}, 95 \%){ }^{1}$ 
2

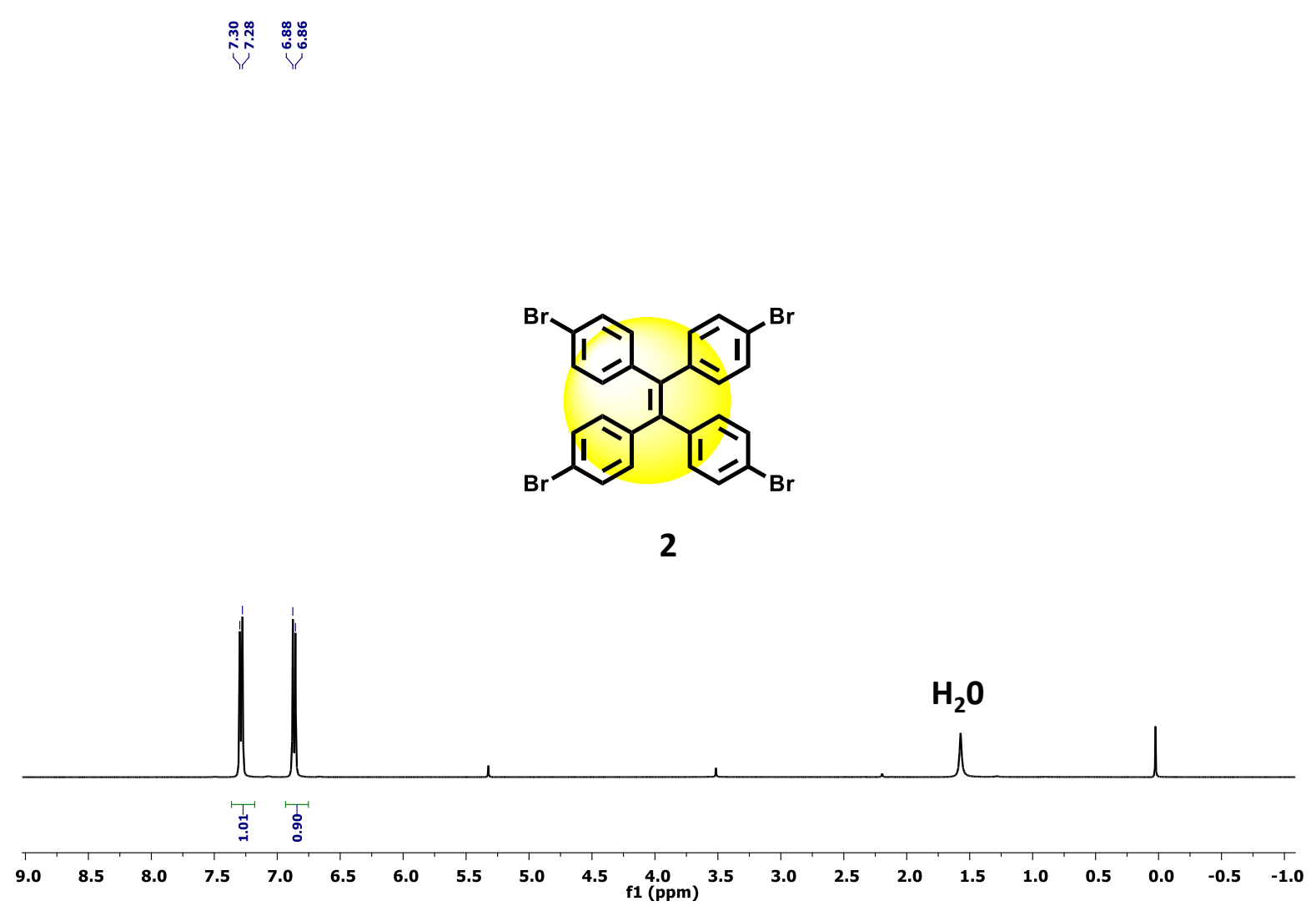

56

${ }^{1} \mathrm{H}$ NMR $\left(400 \mathrm{MHz}, \mathrm{CDCl}_{3}\right) \delta(p p m)=7.287(\mathrm{~d}, J=8.4 \mathrm{~Hz}, 8 \mathrm{H}), 6.865(\mathrm{~d}, J=8.4 \mathrm{~Hz}, 8 \mathrm{H})$

(Figure S1); MS m/z [M] ${ }^{+}$Calcd. for $\mathrm{C}_{26} \mathrm{H}_{16} \mathrm{Br}_{4}$ 647.4679; Found (HRMS-ESI) 647.4634.

Figure S1: ${ }^{1} \mathrm{H}$ NMR spectroscopy of Tetrakis (4-bromophenyl) ethylene (2)

Synthesis of 1, 1, 2, 2-Tetrakis (4-Formyl-(1, 1'-Biphenyl))-Ethane (TFBE) (3).

Tetrakis (4-bromophenyl) ethylene (2) (684 mg, $1 \mathrm{mmol}$ ), 4-formylphenylboronic acid (900 mg, $6 \mathrm{mmol}), \mathrm{K}_{2} \mathrm{CO}_{3}(1.66 \mathrm{~g}, 12 \mathrm{mmol}), \mathrm{Pd}\left(\mathrm{PPh}_{3}\right)_{4}$ catalyst $(10 \mathrm{mg})$ were taken in a Schlenk tube and degassed for $1 \mathrm{hr}$. Toluene $(80 \mathrm{~mL})$, water $(15 \mathrm{~mL})$ and tetra butyl ammonium chloride $(1 \mathrm{~mL})$ were added to the mixture. Then the solution was again degassed by freeze-pump-thaw cycle for 3 times to remove dissolved oxygen. The entire solution was then stirred at $85{ }^{\circ} \mathrm{C}$ for $1 \mathrm{~d}$. After cooling to room temperature, the reaction mixture was mixed with water and the organic layer was extracted by $\mathrm{CH}_{2} \mathrm{Cl}_{2}$ and precipitated out using 
$66 \mathrm{CH}_{3} \mathrm{OH}$ to get a crude product. Recrystallization with $\mathrm{CHCl}_{3}$ and diethyl ether to obtain a

67 yellowish green solid (535 $\mathrm{mg}, 71 \%)^{1}$

$68{ }^{1} \mathrm{H}$ NMR $\left(400 \mathrm{MHz}, \mathrm{CDCl}_{3}\right): \delta(\mathrm{ppm})=10.037(\mathrm{~s}, 4 \mathrm{H}), 7.922(\mathrm{~d}, J=8.4 \mathrm{~Hz}, 8 \mathrm{H}), 7.736(\mathrm{~d}, J$ $69=8.0 \mathrm{~Hz}, 8 \mathrm{H}), 7.48(\mathrm{~d}, J=8.0 \mathrm{~Hz}, 8 \mathrm{H}), 7.235(\mathrm{~d}, J=8.0 \mathrm{~Hz}, 8 \mathrm{H})$ (Figure S2).

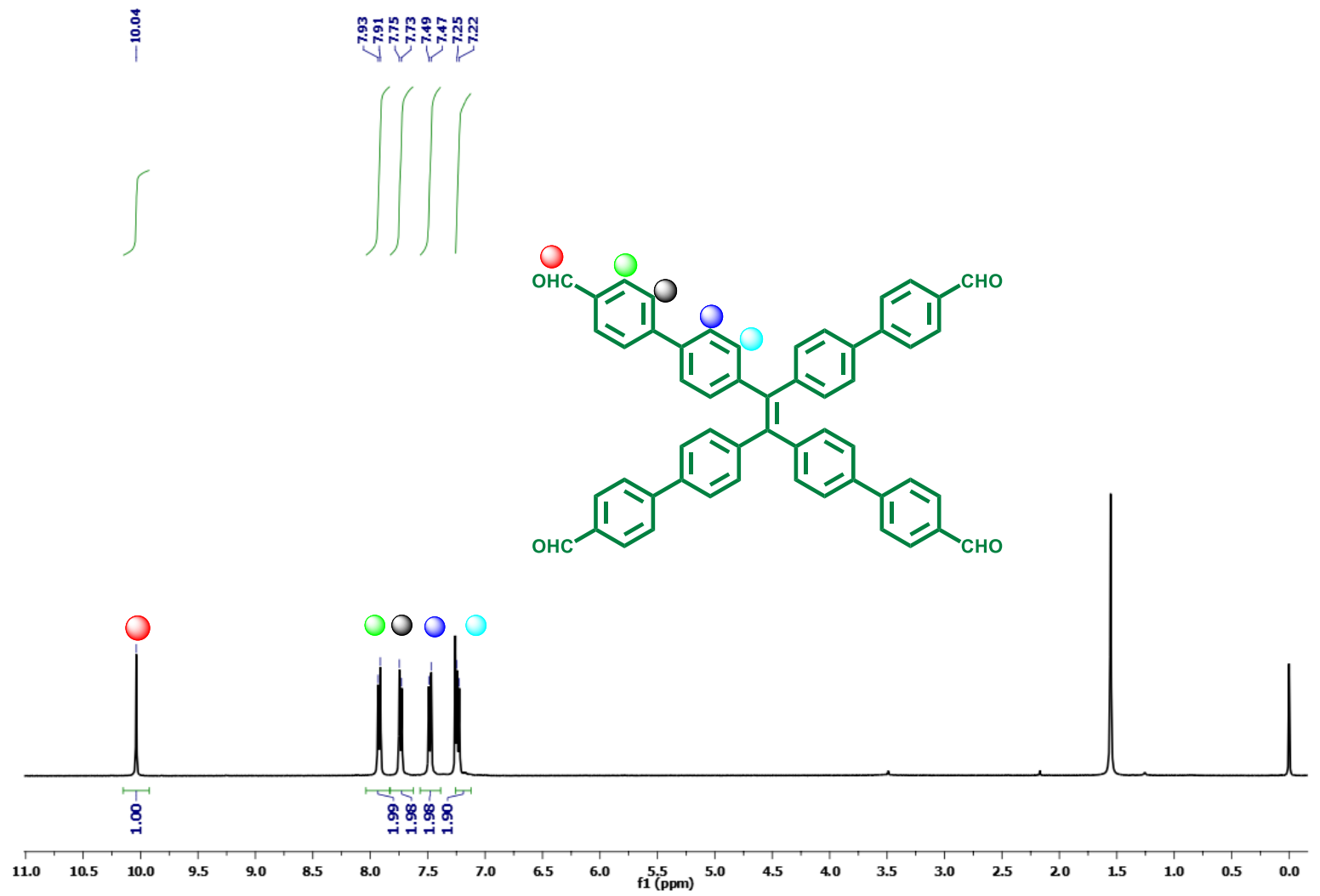

Figure S2: ${ }^{1} \mathrm{H}$ NMR spectroscopy of TFBE (3) 


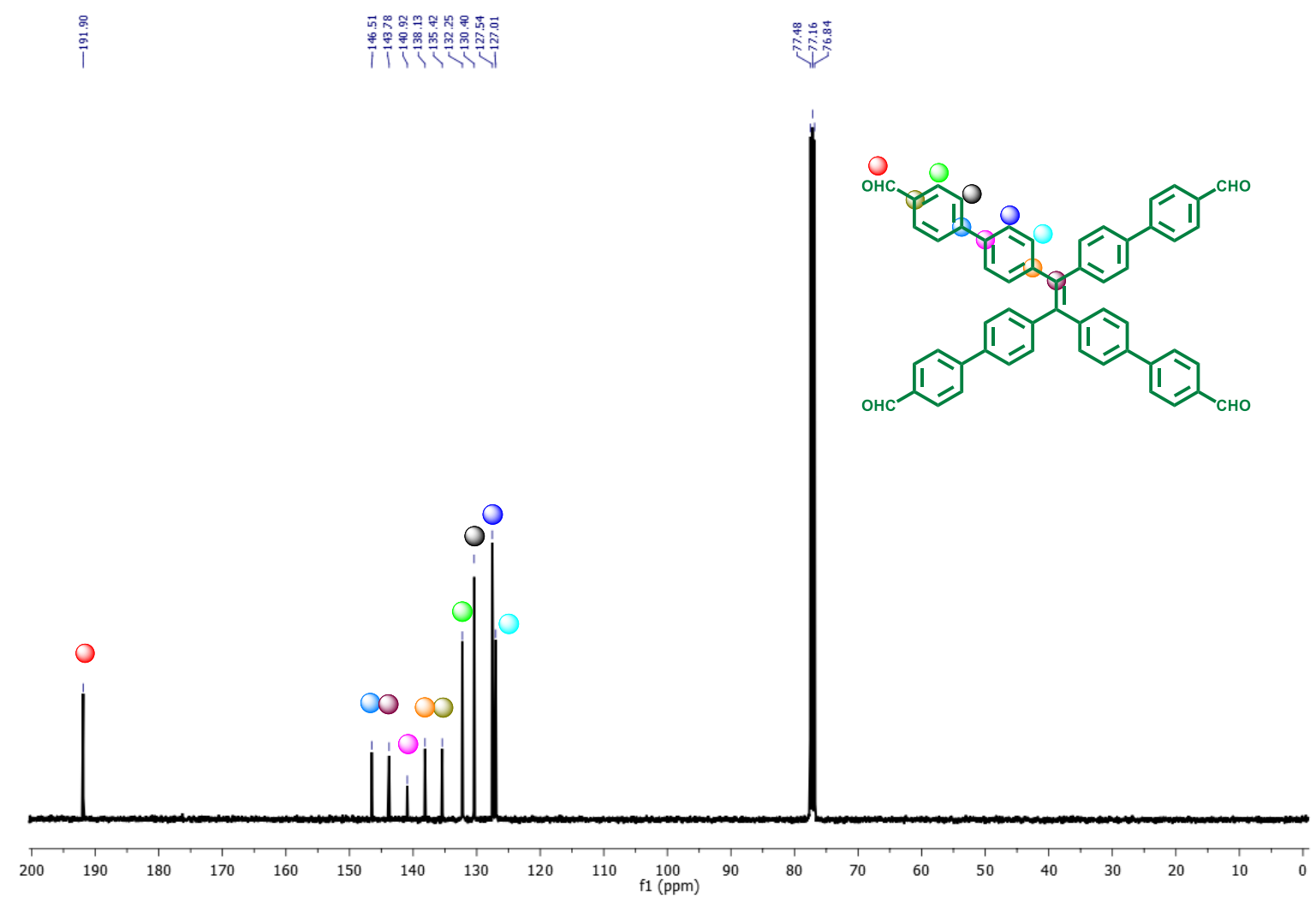

Figure S3: ${ }^{13} \mathrm{C}$ NMR spectroscopy of TFBE (3) aldehyde. Different color ball indicates 73 different peak position with respect to different "C" $\mathrm{C}$ " atom.

$74{ }^{13} \mathrm{C}$ NMR $\left(100 \mathrm{MHz}, \mathrm{CDCl}_{3}\right): \delta(\mathrm{ppm})=191.9,146.51,143.78,140.92,138.13,135.42$, 75 132.25, 130.40, 127.54, 127.01 (Figure S3). MS (MALDI-TOF): m/z calcd. for $\mathrm{C}_{54} \mathrm{H}_{36} \mathrm{O}_{4}$ $76[\mathrm{M}]^{+} 748.2614$, found 748.2694 . 
Fractional atomic coordinates for the unit cell of PT-COF with eclipsed-AA stacking:

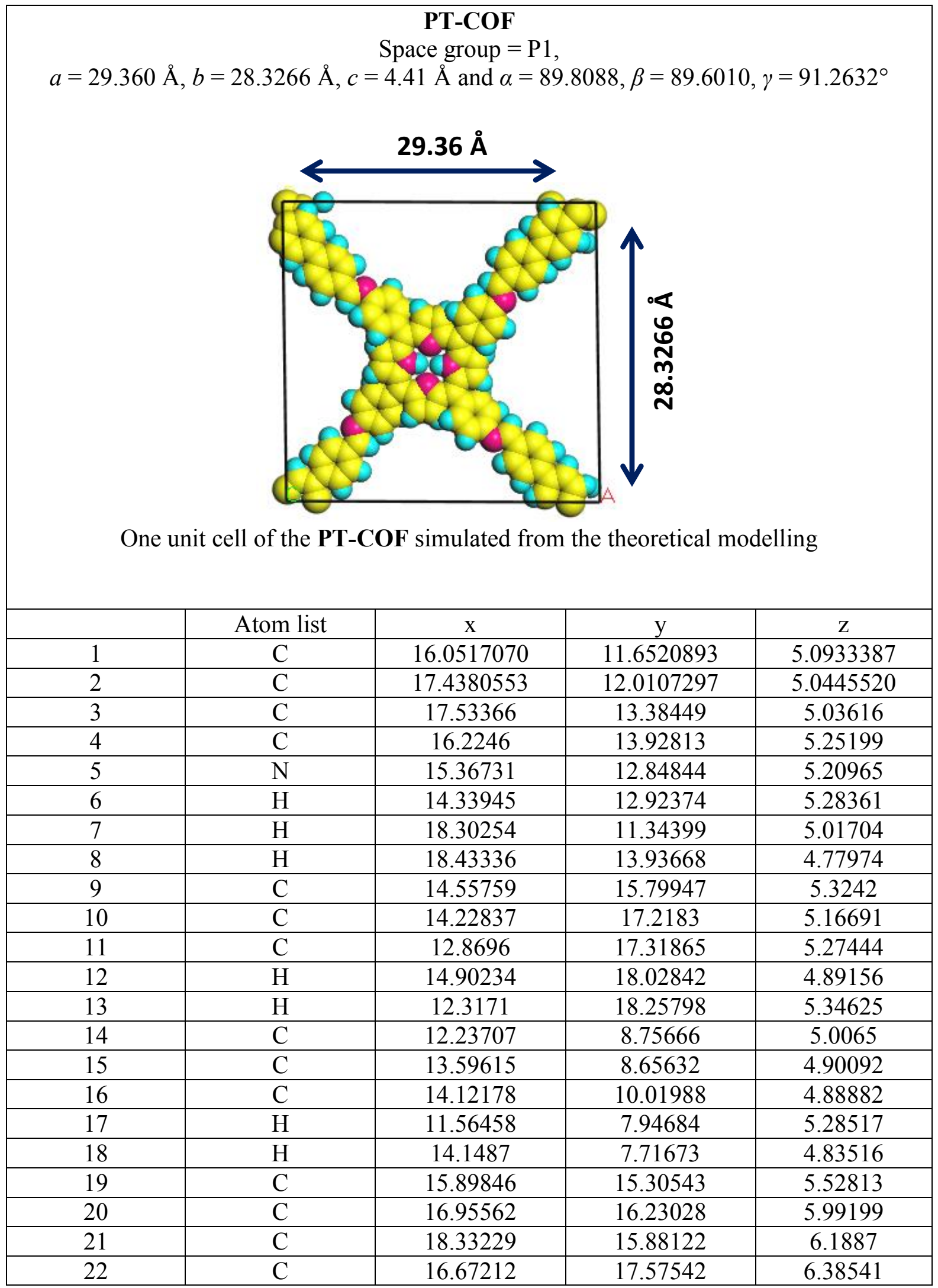




\begin{tabular}{|c|c|c|c|c|}
\hline 23 & $\mathrm{C}$ & 19.33399 & 16.82594 & 6.3454 \\
\hline 24 & $\mathrm{H}$ & 18.64624 & 14.83477 & 6.26149 \\
\hline 25 & $\mathrm{C}$ & 17.65367 & 18.53852 & 6.53079 \\
\hline 26 & $\mathrm{H}$ & 15.65307 & 17.90335 & 6.62524 \\
\hline 27 & $\mathrm{C}$ & 19.02744 & 18.20533 & 6.40726 \\
\hline 28 & $\mathrm{H}$ & 20.38196 & 16.49382 & 6.4459 \\
\hline 29 & $\mathrm{H}$ & 17.35271 & 19.56571 & 6.78507 \\
\hline 30 & $\mathrm{C}$ & 15.51324 & 10.32492 & 4.98093 \\
\hline 31 & $\mathrm{C}$ & 16.50472 & 9.19326 & 4.98035 \\
\hline 32 & $\mathrm{C}$ & 16.29646 & 7.98334 & 5.68073 \\
\hline 33 & $\mathrm{C}$ & 17.72673 & 9.28109 & 4.26065 \\
\hline 34 & $\mathrm{C}$ & 17.2061 & 6.9237 & 5.62375 \\
\hline 35 & $\mathrm{H}$ & 15.40244 & 7.83483 & 6.29857 \\
\hline 36 & $\mathrm{C}$ & 18.72742 & 8.31237 & 4.36973 \\
\hline 37 & $\mathrm{H}$ & 17.9342 & 10.10993 & 3.58895 \\
\hline 38 & $\mathrm{C}$ & 18.4743 & 7.10347 & 5.04006 \\
\hline 39 & $\mathrm{H}$ & 16.94324 & 5.95735 & 6.08859 \\
\hline 40 & $\mathrm{H}$ & 19.70207 & 8.50835 & 3.88499 \\
\hline 41 & $\mathrm{C}$ & 10.24227 & 12.04653 & 4.91497 \\
\hline 42 & $\mathrm{C}$ & 10.41314 & 14.3223 & 5.07792 \\
\hline 43 & $\mathrm{C}$ & 9.02712 & 13.96225 & 5.1269 \\
\hline 44 & $\mathrm{H}$ & 8.03393 & 12.03232 & 5.38723 \\
\hline 45 & $\mathrm{H}$ & 8.16265 & 14.62899 & 5.1552 \\
\hline 46 & $\mathrm{C}$ & 10.95158 & 15.65032 & 5.18896 \\
\hline 47 & $\mathrm{C}$ & 12.34364 & 15.95479 & 5.28107 \\
\hline 48 & $\mathrm{C}$ & 11.90754 & 10.17463 & 4.84375 \\
\hline 49 & $\mathrm{C}$ & 10.56662 & 10.6695 & 4.6363 \\
\hline 50 & $\mathrm{C}$ & 9.50843 & 9.74945 & 4.16522 \\
\hline 51 & $\mathrm{C}$ & 9.78393 & 8.39897 & 3.78624 \\
\hline 52 & $\mathrm{C}$ & 8.13836 & 10.11088 & 3.94334 \\
\hline 53 & $\mathrm{C}$ & 8.7956 & 7.44516 & 3.62447 \\
\hline 54 & $\mathrm{H}$ & 10.80432 & 8.05782 & 3.571 \\
\hline 55 & $\mathrm{C}$ & 7.13019 & 9.17459 & 3.77102 \\
\hline 56 & $\mathrm{H}$ & 7.83718 & 11.16098 & 3.86294 \\
\hline 57 & $\mathrm{C}$ & 7.42192 & 7.7907 & 3.71697 \\
\hline 58 & $\mathrm{H}$ & 9.09377 & 6.41433 & 3.38112 \\
\hline 59 & $\mathrm{H}$ & 6.08829 & 9.51797 & 3.64886 \\
\hline 60 & $\mathrm{C}$ & 9.96274 & 16.78312 & 5.19267 \\
\hline 61 & $\mathrm{C}$ & 8.74096 & 16.69841 & 5.91218 \\
\hline 62 & $\mathrm{C}$ & 10.17596 & 17.99646 & 4.49755 \\
\hline 63 & $\mathrm{C}$ & 7.74954 & 17.67709 & 5.81733 \\
\hline 64 & $\mathrm{H}$ & 8.52797 & 15.86444 & 6.57607 \\
\hline 65 & $\mathrm{C}$ & 9.27681 & 19.06436 & 4.56898 \\
\hline 66 & $\mathrm{H}$ & 11.06653 & 18.13984 & 3.87406 \\
\hline 67 & $\mathrm{C}$ & 8.01112 & 18.89282 & 5.16027 \\
\hline 68 & $\mathrm{H}$ & 6.77539 & 17.48536 & 6.30403 \\
\hline 69 & $\mathrm{H}$ & 9.54732 & 20.03129 & 4.1096 \\
\hline 70 & $\mathrm{~N}$ & 11.09869 & 13.1268 & 4.96025 \\
\hline
\end{tabular}




\begin{tabular}{|c|c|c|c|c|}
\hline 71 & $\mathrm{~N}$ & 13.06208 & 10.90359 & 4.84804 \\
\hline 72 & $\mathrm{~N}$ & 13.40245 & 15.07078 & 5.31815 \\
\hline 73 & $\mathrm{C}$ & 8.93239 & 12.58819 & 5.13276 \\
\hline 74 & $\mathrm{H}$ & 12.12653 & 13.05234 & 4.8859 \\
\hline 75 & $\mathrm{C}$ & 19.80995 & 20.40227 & 6.54133 \\
\hline 76 & $\mathrm{H}$ & 18.76671 & 20.81614 & 6.49274 \\
\hline 77 & $\mathrm{C}$ & 20.67345 & 6.29474 & 4.97996 \\
\hline 78 & $\mathrm{H}$ & 21.0861 & 7.33285 & 4.8738 \\
\hline 79 & $\mathrm{C}$ & 5.8228 & 19.72974 & 5.26214 \\
\hline 80 & $\mathrm{H}$ & 5.39705 & 18.69641 & 5.35757 \\
\hline 81 & $\mathrm{C}$ & 6.61824 & 5.60047 & 3.55792 \\
\hline 82 & $\mathrm{H}$ & 7.65712 & 5.17733 & 3.62583 \\
\hline 83 & $\mathrm{C}$ & 20.85723 & 21.40041 & 6.66583 \\
\hline 84 & $\mathrm{C}$ & 20.53394 & 22.77493 & 6.71306 \\
\hline 85 & $\mathrm{C}$ & 22.22228 & 21.03222 & 6.73687 \\
\hline 86 & $\mathrm{C}$ & 21.51946 & 23.7391 & 6.84147 \\
\hline 87 & $\mathrm{H}$ & 19.47759 & 23.08995 & 6.66017 \\
\hline 88 & $\mathrm{C}$ & 23.20905 & 21.99213 & 6.86313 \\
\hline 89 & $\mathrm{H}$ & 22.48715 & 19.96728 & 6.70213 \\
\hline 90 & $\mathrm{C}$ & 22.8917 & 23.37299 & 6.93963 \\
\hline 91 & $\mathrm{H}$ & 21.20776 & 24.79639 & 6.87347 \\
\hline 92 & $\mathrm{H}$ & 24.25438 & 21.64255 & 6.91133 \\
\hline 93 & $\mathrm{C}$ & 21.66454 & 5.22457 & 4.99089 \\
\hline 94 & $\mathrm{C}$ & 23.0387 & 5.53662 & 4.90034 \\
\hline 95 & $\mathrm{C}$ & 21.29587 & 3.86813 & 5.09197 \\
\hline 96 & $\mathrm{C}$ & 24.00237 & 4.53495 & 4.90053 \\
\hline 97 & $\mathrm{H}$ & 23.36141 & 6.58528 & 4.81252 \\
\hline 98 & $\mathrm{C}$ & 22.25984 & 2.86504 & 5.09061 \\
\hline 99 & $\mathrm{H}$ & 20.2275 & 3.60753 & 5.15813 \\
\hline 100 & $\mathrm{C}$ & 23.64259 & 3.1671 & 4.97879 \\
\hline 101 & $\mathrm{H}$ & 25.05783 & 4.84422 & 4.82286 \\
\hline 102 & $\mathrm{H}$ & 21.90519 & 1.82457 & 5.17023 \\
\hline 103 & $\mathrm{C}$ & 4.85044 & 20.81505 & 5.28985 \\
\hline 104 & $\mathrm{C}$ & 3.47147 & 20.52771 & 5.4007 \\
\hline 105 & $\mathrm{C}$ & 5.24374 & 22.16676 & 5.21101 \\
\hline 106 & $\mathrm{C}$ & 2.52763 & 21.54621 & 5.43407 \\
\hline 107 & $\mathrm{H}$ & 3.12856 & 19.48323 & 5.46763 \\
\hline 108 & $\mathrm{C}$ & 4.30078 & 23.18639 & 5.24614 \\
\hline 109 & $\mathrm{H}$ & 6.31473 & 22.40859 & 5.12495 \\
\hline 110 & $\mathrm{C}$ & 2.91315 & 22.90872 & 5.36693 \\
\hline 111 & $\mathrm{H}$ & 1.46714 & 21.25539 & 5.52188 \\
\hline 112 & $\mathrm{H}$ & 4.67146 & 24.22237 & 5.17823 \\
\hline 113 & $\mathrm{C}$ & 5.56909 & 4.61072 & 3.40219 \\
\hline 114 & $\mathrm{C}$ & 5.88567 & 3.23279 & 3.35126 \\
\hline 115 & $\mathrm{C}$ & 4.20522 & 4.98191 & 3.29974 \\
\hline 116 & $\mathrm{C}$ & 4.90132 & 2.27333 & 3.19417 \\
\hline 117 & $\mathrm{H}$ & 6.9377 & 2.90994 & 3.43205 \\
\hline 118 & $\mathrm{C}$ & 3.21812 & 4.02756 & 3.14284 \\
\hline
\end{tabular}




\begin{tabular}{|c|c|c|c|c|}
\hline 119 & $\mathrm{H}$ & 3.94159 & 6.04689 & 3.34306 \\
\hline 120 & $\mathrm{C}$ & 3.52911 & 2.64246 & 3.06791 \\
\hline 121 & $\mathrm{H}$ & 5.20953 & 1.21492 & 3.16333 \\
\hline 122 & $\mathrm{H}$ & 2.17486 & 4.38216 & 3.07279 \\
\hline 123 & $\mathrm{~N}$ & 19.40325 & 6.05555 & 5.09334 \\
\hline 124 & $\mathrm{~N}$ & 20.06548 & 19.13227 & 6.4982 \\
\hline 125 & $\mathrm{~N}$ & 7.0956 & 19.95245 & 5.13237 \\
\hline 126 & $\mathrm{~N}$ & 6.37671 & 6.8753 & 3.603 \\
\hline 127 & $\mathrm{C}$ & 23.93381 & 24.37262 & 7.16834 \\
\hline 128 & $\mathrm{C}$ & 25.29943 & 23.98654 & 7.30572 \\
\hline 129 & $\mathrm{C}$ & 23.62291 & 25.74589 & 7.34475 \\
\hline 130 & $\mathrm{C}$ & 26.29626 & 24.90943 & 7.5356 \\
\hline 131 & $\mathrm{H}$ & 25.60086 & 22.93712 & 7.17419 \\
\hline 132 & $\mathrm{C}$ & 24.60282 & 26.68644 & 7.60234 \\
\hline 133 & $\mathrm{H}$ & 22.58795 & 26.11799 & 7.25596 \\
\hline 134 & $\mathrm{C}$ & 25.98888 & 26.3033 & 7.60917 \\
\hline 135 & $\mathrm{H}$ & 27.33987 & 24.57189 & 7.62693 \\
\hline 136 & $\mathrm{H}$ & 24.33492 & 27.73675 & 7.74519 \\
\hline 137 & $\mathrm{C}$ & 24.67664 & 2.10357 & 4.94112 \\
\hline 138 & $\mathrm{C}$ & 24.33424 & 0.73587 & 4.9017 \\
\hline 139 & $\mathrm{C}$ & 26.05387 & 2.42527 & 4.94034 \\
\hline 140 & $\mathrm{C}$ & 24.6806 & 28.05455 & 4.92063 \\
\hline 141 & $\mathrm{H}$ & 23.28213 & 0.41635 & 4.88238 \\
\hline 142 & $\mathrm{C}$ & 27.02513 & 1.43805 & 5.0225 \\
\hline 143 & $\mathrm{H}$ & 26.38884 & 3.46861 & 4.95225 \\
\hline 144 & $\mathrm{C}$ & 26.66925 & 0.07796 & 5.05255 \\
\hline 145 & $\mathrm{H}$ & 24.36832 & 27.01711 & 4.81486 \\
\hline 146 & $\mathrm{H}$ & 28.07705 & 1.72814 & 5.04845 \\
\hline 147 & $\mathrm{C}$ & 1.90598 & 23.9918 & 5.40577 \\
\hline 148 & $\mathrm{C}$ & 2.2815 & 25.35517 & 5.42372 \\
\hline 149 & $\mathrm{C}$ & 0.52053 & 23.69585 & 5.40304 \\
\hline 150 & $\mathrm{C}$ & 1.33031 & 26.37122 & 5.40499 \\
\hline 151 & $\mathrm{H}$ & 3.33981 & 25.65652 & 5.4327 \\
\hline 152 & $\mathrm{C}$ & -0.43022 & 24.69893 & 5.32303 \\
\hline 153 & $\mathrm{H}$ & 0.16683 & 22.65684 & 5.41494 \\
\hline 154 & $\mathrm{C}$ & -0.04481 & 26.05768 & 5.31249 \\
\hline 155 & $\mathrm{H}$ & 1.65848 & 27.40853 & 5.47407 \\
\hline 156 & $\mathrm{H}$ & 27.87146 & 24.42863 & 5.284 \\
\hline 157 & $\mathrm{C}$ & 2.49211 & 1.64818 & 2.83139 \\
\hline 158 & $\mathrm{C}$ & 1.12212 & 2.02626 & 2.67332 \\
\hline 159 & $\mathrm{C}$ & 2.79209 & 0.26229 & 2.69088 \\
\hline 160 & $\mathrm{C}$ & 0.13415 & 1.11399 & 2.40148 \\
\hline 161 & $\mathrm{H}$ & 0.82018 & 3.07844 & 2.78241 \\
\hline 162 & $\mathrm{C}$ & 1.1996 & 27.64567 & 2.42392 \\
\hline 163 & $\mathrm{H}$ & 3.20093 & 28.21512 & 2.81129 \\
\hline 164 & $\mathrm{C}$ & -0.19104 & 28.02472 & 2.29727 \\
\hline 165 & $\mathrm{H}$ & 28.45568 & 1.45059 & 2.28595 \\
\hline 166 & $\mathrm{H}$ & 1.46881 & 26.58871 & 2.32127 \\
\hline
\end{tabular}




\begin{tabular}{|c|c|c|c|c|}
\hline 167 & $\mathrm{C}$ & 28.26772 & 27.02478 & 5.14427 \\
\hline 168 & $\mathrm{C}$ & 27.11399 & 27.44992 & 5.32537 \\
\hline 169 & $\mathrm{C}$ & 28.19622 & 27.08839 & 2.19482 \\
\hline 170 & $\mathrm{C}$ & 26.92169 & 27.31918 & 7.54322 \\
\hline 171 & $\mathrm{C}$ & 16.11966 & 11.68616 & 14.85069 \\
\hline 172 & $\mathrm{C}$ & 17.50601 & 12.0448 & 14.80191 \\
\hline 173 & $\mathrm{C}$ & 17.60161 & 13.41856 & 14.79351 \\
\hline 174 & $\mathrm{C}$ & 16.29255 & 13.9622 & 15.00935 \\
\hline 175 & $\mathrm{~N}$ & 15.43526 & 12.88251 & 14.967 \\
\hline 176 & $\mathrm{H}$ & 14.4074 & 12.95781 & 15.04096 \\
\hline 177 & $\mathrm{H}$ & 18.37049 & 11.37806 & 14.77439 \\
\hline 178 & $\mathrm{H}$ & 18.50131 & 13.97075 & 14.53709 \\
\hline 179 & $\mathrm{C}$ & 14.62554 & 15.83354 & 15.08155 \\
\hline 180 & $\mathrm{C}$ & 14.29632 & 17.25237 & 14.92426 \\
\hline 181 & $\mathrm{C}$ & 12.93755 & 17.35272 & 15.03179 \\
\hline 182 & $\mathrm{H}$ & 14.97029 & 18.06249 & 14.64891 \\
\hline 183 & $\mathrm{H}$ & 12.38505 & 18.29205 & 15.1036 \\
\hline 184 & $\mathrm{C}$ & 12.30502 & 8.79073 & 14.76385 \\
\hline 185 & $\mathrm{C}$ & 13.6641 & 8.69039 & 14.65828 \\
\hline 186 & $\mathrm{C}$ & 14.18973 & 10.05394 & 14.64618 \\
\hline 187 & $\mathrm{H}$ & 11.63253 & 7.98091 & 15.04252 \\
\hline 188 & $\mathrm{H}$ & 14.21665 & 7.7508 & 14.59251 \\
\hline 189 & $\mathrm{C}$ & 15.96641 & 15.3395 & 15.28548 \\
\hline 190 & $\mathrm{C}$ & 17.02357 & 16.26435 & 15.74935 \\
\hline 191 & $\mathrm{C}$ & 18.40024 & 15.91528 & 15.94605 \\
\hline 192 & $\mathrm{C}$ & 16.74007 & 17.60949 & 16.14276 \\
\hline 193 & $\mathrm{C}$ & 19.40194 & 16.86001 & 16.10276 \\
\hline 194 & $\mathrm{H}$ & 18.7142 & 14.86884 & 16.01884 \\
\hline 195 & $\mathrm{C}$ & 17.72162 & 18.57258 & 16.28815 \\
\hline 196 & $\mathrm{H}$ & 15.72102 & 17.93742 & 16.3826 \\
\hline 197 & $\mathrm{C}$ & 19.09539 & 18.2394 & 16.16462 \\
\hline 198 & $\mathrm{H}$ & 20.44991 & 16.52789 & 16.20326 \\
\hline 199 & $\mathrm{H}$ & 17.42067 & 19.59978 & 16.54242 \\
\hline 200 & $\mathrm{C}$ & 15.58119 & 10.35899 & 14.73829 \\
\hline 201 & $\mathrm{C}$ & 16.57267 & 9.22733 & 14.7377 \\
\hline 202 & $\mathrm{C}$ & 16.36441 & 8.01741 & 15.43809 \\
\hline 203 & $\mathrm{C}$ & 17.79468 & 9.31516 & 14.018 \\
\hline 204 & $\mathrm{C}$ & 17.27405 & 6.95777 & 15.3811 \\
\hline 205 & $\mathrm{H}$ & 15.47039 & 7.8689 & 16.05573 \\
\hline 206 & $\mathrm{C}$ & 18.79537 & 8.34644 & 14.12709 \\
\hline 207 & $\mathrm{H}$ & 18.00215 & 10.14399 & 13.3463 \\
\hline 208 & $\mathrm{C}$ & 18.54225 & 7.13754 & 14.79742 \\
\hline 209 & $\mathrm{H}$ & 17.01119 & 5.99142 & 15.84594 \\
\hline 210 & $\mathrm{H}$ & 19.77002 & 8.54241 & 13.64234 \\
\hline 211 & $\mathrm{C}$ & 10.30993 & 12.08059 & 14.67233 \\
\hline 212 & $\mathrm{C}$ & 10.48109 & 14.35637 & 14.83528 \\
\hline 213 & $\mathrm{C}$ & 9.09507 & 13.99631 & 14.88426 \\
\hline 214 & $\mathrm{H}$ & 8.10188 & 12.06638 & 15.14458 \\
\hline
\end{tabular}




\begin{tabular}{|c|c|c|c|c|}
\hline 215 & $\mathrm{H}$ & 8.2306 & 14.66306 & 14.91255 \\
\hline 216 & $\mathrm{C}$ & 11.01953 & 15.68439 & 14.94631 \\
\hline 217 & $\mathrm{C}$ & 12.41159 & 15.98886 & 15.03842 \\
\hline 218 & $\mathrm{C}$ & 11.97549 & 10.2087 & 14.6011 \\
\hline 219 & $\mathrm{C}$ & 10.63457 & 10.70357 & 14.39366 \\
\hline 220 & $\mathrm{C}$ & 9.57638 & 9.78351 & 13.92257 \\
\hline 221 & $\mathrm{C}$ & 9.85188 & 8.43304 & 13.5436 \\
\hline 222 & $\mathrm{C}$ & 8.20631 & 10.14495 & 13.70069 \\
\hline 223 & $\mathrm{C}$ & 8.86355 & 7.47923 & 13.38182 \\
\hline 224 & $\mathrm{H}$ & 10.87227 & 8.09188 & 13.32835 \\
\hline 225 & $\mathrm{C}$ & 7.19814 & 9.20866 & 13.52838 \\
\hline 226 & $\mathrm{H}$ & 7.90513 & 11.19505 & 13.62029 \\
\hline 227 & $\mathrm{C}$ & 7.48987 & 7.82477 & 13.47432 \\
\hline 228 & $\mathrm{H}$ & 9.16172 & 6.44839 & 13.13847 \\
\hline 229 & $\mathrm{H}$ & 6.15624 & 9.55204 & 13.40621 \\
\hline 230 & $\mathrm{C}$ & 10.03069 & 16.81719 & 14.95002 \\
\hline 231 & $\mathrm{C}$ & 8.80891 & 16.73247 & 15.66953 \\
\hline 232 & $\mathrm{C}$ & 10.24391 & 18.03053 & 14.25491 \\
\hline 233 & $\mathrm{C}$ & 7.81749 & 17.71116 & 15.57469 \\
\hline 234 & $\mathrm{H}$ & 8.59592 & 15.89851 & 16.33342 \\
\hline 235 & $\mathrm{C}$ & 9.34476 & 19.09843 & 14.32633 \\
\hline 236 & $\mathrm{H}$ & 11.13448 & 18.17391 & 13.63141 \\
\hline 237 & $\mathrm{C}$ & 8.07907 & 18.92689 & 14.91763 \\
\hline 238 & $\mathrm{H}$ & 6.84335 & 17.51943 & 16.06139 \\
\hline 239 & $\mathrm{H}$ & 9.61527 & 20.06536 & 13.86696 \\
\hline 240 & $\mathrm{~N}$ & 11.16664 & 13.16087 & 14.7176 \\
\hline 241 & $\mathrm{~N}$ & 13.13003 & 10.93766 & 14.60539 \\
\hline 242 & $\mathrm{~N}$ & 13.4704 & 15.10485 & 15.0755 \\
\hline 243 & $\mathrm{C}$ & 9.00034 & 12.62226 & 14.89011 \\
\hline 244 & $\mathrm{H}$ & 12.19448 & 13.08641 & 14.64325 \\
\hline 245 & $\mathrm{C}$ & 19.8779 & 20.43634 & 16.29868 \\
\hline 246 & $\mathrm{H}$ & 18.83467 & 20.85021 & 16.25009 \\
\hline 247 & $\mathrm{C}$ & 20.7414 & 6.3288 & 14.73731 \\
\hline 248 & $\mathrm{H}$ & 21.15405 & 7.36692 & 14.63115 \\
\hline 249 & $\mathrm{C}$ & 5.89075 & 19.76381 & 15.01949 \\
\hline 250 & $\mathrm{H}$ & 5.465 & 18.73048 & 15.11512 \\
\hline 251 & $\mathrm{C}$ & 6.6862 & 5.63454 & 13.31528 \\
\hline 252 & $\mathrm{H}$ & 7.72507 & 5.21139 & 13.38319 \\
\hline 253 & $\mathrm{C}$ & 20.92518 & 21.43448 & 16.42319 \\
\hline 254 & $\mathrm{C}$ & 20.60189 & 22.809 & 16.47041 \\
\hline 255 & $\mathrm{C}$ & 22.29023 & 21.06629 & 16.49422 \\
\hline 256 & $\mathrm{C}$ & 21.58741 & 23.77317 & 16.59882 \\
\hline 257 & $\mathrm{H}$ & 19.54554 & 23.12401 & 16.41753 \\
\hline 258 & $\mathrm{C}$ & 23.277 & 22.0262 & 16.62048 \\
\hline 259 & $\mathrm{H}$ & 22.5551 & 20.00135 & 16.45949 \\
\hline 260 & $\mathrm{C}$ & 22.95965 & 23.40705 & 16.69698 \\
\hline 261 & $\mathrm{H}$ & 21.27571 & 24.83046 & 16.63082 \\
\hline 262 & $\mathrm{H}$ & 24.32233 & 21.67662 & 16.66868 \\
\hline
\end{tabular}




\begin{tabular}{|c|c|c|c|c|}
\hline 263 & $\mathrm{C}$ & 21.73249 & 5.25864 & 14.74824 \\
\hline 264 & $\mathrm{C}$ & 23.10665 & 5.57069 & 14.65769 \\
\hline 265 & $\mathrm{C}$ & 21.36382 & 3.9022 & 14.84933 \\
\hline 266 & $\mathrm{C}$ & 24.07032 & 4.56902 & 14.65789 \\
\hline 267 & $\mathrm{H}$ & 23.42936 & 6.61935 & 14.56988 \\
\hline 268 & $\mathrm{C}$ & 22.32779 & 2.89911 & 14.84796 \\
\hline 269 & $\mathrm{H}$ & 20.29545 & 3.6416 & 14.91548 \\
\hline 270 & $\mathrm{C}$ & 23.71054 & 3.20117 & 14.73614 \\
\hline 271 & $\mathrm{H}$ & 25.12578 & 4.87829 & 14.58022 \\
\hline 272 & $\mathrm{H}$ & 21.97314 & 1.85863 & 14.92758 \\
\hline 273 & $\mathrm{C}$ & 4.91839 & 20.84912 & 15.04721 \\
\hline 274 & $\mathrm{C}$ & 3.53942 & 20.56178 & 15.15805 \\
\hline 275 & $\mathrm{C}$ & 5.31169 & 22.20083 & 14.96837 \\
\hline 276 & $\mathrm{C}$ & 2.59558 & 21.58027 & 15.19142 \\
\hline 277 & $\mathrm{H}$ & 3.19651 & 19.5173 & 15.22498 \\
\hline 278 & $\mathrm{C}$ & 4.36873 & 23.22046 & 15.00349 \\
\hline 279 & $\mathrm{H}$ & 6.38268 & 22.44266 & 14.88231 \\
\hline 280 & $\mathrm{C}$ & 2.9811 & 22.94278 & 15.12429 \\
\hline 281 & $\mathrm{H}$ & 1.53509 & 21.28945 & 15.27924 \\
\hline 282 & $\mathrm{H}$ & 4.73941 & 24.25644 & 14.93558 \\
\hline 283 & $\mathrm{C}$ & 5.63705 & 4.64479 & 13.15955 \\
\hline 284 & $\mathrm{C}$ & 5.95362 & 3.26685 & 13.10861 \\
\hline 285 & $\mathrm{C}$ & 4.27317 & 5.01598 & 13.0571 \\
\hline 286 & $\mathrm{C}$ & 4.96927 & 2.3074 & 12.95152 \\
\hline 287 & $\mathrm{H}$ & 7.00565 & 2.94401 & 13.18941 \\
\hline 288 & $\mathrm{C}$ & 3.28607 & 4.06163 & 12.9002 \\
\hline 289 & $\mathrm{H}$ & 4.00954 & 6.08096 & 13.10042 \\
\hline 290 & $\mathrm{C}$ & 3.59706 & 2.67653 & 12.82526 \\
\hline 291 & $\mathrm{H}$ & 5.27748 & 1.24898 & 12.92088 \\
\hline 292 & $\mathrm{H}$ & 2.24281 & 4.41623 & 12.83014 \\
\hline 293 & $\mathrm{~N}$ & 19.4712 & 6.08962 & 14.85069 \\
\hline 294 & $\mathrm{~N}$ & 20.13344 & 19.16605 & 16.25556 \\
\hline 295 & $\mathrm{~N}$ & 7.16355 & 19.98652 & 14.88972 \\
\hline 296 & $\mathrm{~N}$ & 6.44466 & 6.90936 & 13.36035 \\
\hline 297 & $\mathrm{C}$ & 24.00176 & 24.40669 & 16.92569 \\
\hline 298 & $\mathrm{C}$ & 25.36738 & 24.0206 & 17.06308 \\
\hline 299 & $\mathrm{C}$ & 23.69086 & 25.77996 & 17.1021 \\
\hline 300 & $\mathrm{C}$ & 26.36421 & 24.9435 & 17.29296 \\
\hline 301 & $\mathrm{H}$ & 25.66881 & 22.97118 & 16.93155 \\
\hline 302 & $\mathrm{C}$ & 24.67077 & 26.72051 & 17.3597 \\
\hline 303 & $\mathrm{H}$ & 22.6559 & 26.15206 & 17.01331 \\
\hline 304 & $\mathrm{C}$ & 26.05683 & 26.33737 & 17.36653 \\
\hline 305 & $\mathrm{H}$ & 27.40782 & 24.60596 & 17.38429 \\
\hline 306 & $\mathrm{H}$ & 24.40287 & 27.77082 & 17.50255 \\
\hline 307 & $\mathrm{C}$ & 24.74459 & 2.13763 & 14.69848 \\
\hline 308 & $\mathrm{C}$ & 24.40219 & 0.76994 & 14.65906 \\
\hline 309 & $\mathrm{C}$ & 26.12182 & 2.45934 & 14.6977 \\
\hline 310 & $\mathrm{C}$ & 24.74855 & 28.08862 & 14.67799 \\
\hline
\end{tabular}




\begin{tabular}{|c|c|c|c|c|}
\hline 311 & $\mathrm{H}$ & 23.35008 & 0.45042 & 14.63974 \\
\hline 312 & $\mathrm{C}$ & 27.09308 & 1.47212 & 14.77985 \\
\hline 313 & $\mathrm{H}$ & 26.45679 & 3.50268 & 14.7096 \\
\hline 314 & $\mathrm{C}$ & 26.7372 & 0.11203 & 14.80991 \\
\hline 315 & $\mathrm{H}$ & 24.43627 & 27.05118 & 14.57222 \\
\hline 316 & $\mathrm{H}$ & 28.145 & 1.76221 & 14.80581 \\
\hline 317 & $\mathrm{C}$ & 1.97393 & 24.02587 & 15.16312 \\
\hline 318 & $\mathrm{C}$ & 2.34945 & 25.38924 & 15.18108 \\
\hline 319 & $\mathrm{C}$ & 0.58848 & 23.72991 & 15.16039 \\
\hline 320 & $\mathrm{C}$ & 1.39826 & 26.40529 & 15.16234 \\
\hline 321 & $\mathrm{H}$ & 3.40776 & 25.69059 & 15.19005 \\
\hline 322 & $\mathrm{C}$ & -0.36227 & 24.733 & 15.08038 \\
\hline 323 & $\mathrm{H}$ & 0.23478 & 22.69091 & 15.1723 \\
\hline 324 & $\mathrm{C}$ & 0.02314 & 26.09175 & 15.06984 \\
\hline 325 & $\mathrm{H}$ & 1.72643 & 27.44259 & 15.23142 \\
\hline 326 & $\mathrm{H}$ & 27.93941 & 24.4627 & 15.04135 \\
\hline 327 & $\mathrm{C}$ & 2.56006 & 1.68225 & 12.58874 \\
\hline 328 & $\mathrm{C}$ & 1.19007 & 2.06033 & 12.43067 \\
\hline 329 & $\mathrm{C}$ & 2.86004 & 0.29636 & 12.44824 \\
\hline 330 & $\mathrm{C}$ & 0.2021 & 1.14805 & 12.15883 \\
\hline 331 & $\mathrm{H}$ & 0.88813 & 3.11251 & 12.53976 \\
\hline 332 & $\mathrm{C}$ & 1.26755 & 27.67974 & 12.18128 \\
\hline 333 & $\mathrm{H}$ & 3.26888 & 28.24919 & 12.56864 \\
\hline 334 & $\mathrm{C}$ & -0.12309 & 28.05878 & 12.05463 \\
\hline 335 & $\mathrm{H}$ & 28.52363 & 1.48466 & 12.04331 \\
\hline 336 & $\mathrm{H}$ & 1.53676 & 26.62278 & 12.07863 \\
\hline 337 & $\mathrm{C}$ & 28.33567 & 27.05884 & 14.90163 \\
\hline 338 & $\mathrm{C}$ & 27.18194 & 27.48399 & 15.08272 \\
\hline 339 & $\mathrm{C}$ & 28.26417 & 27.12246 & 11.95217 \\
\hline 340 & $\mathrm{C}$ & 26.98964 & 27.35325 & 17.30057 \\
\hline
\end{tabular}

80

81 

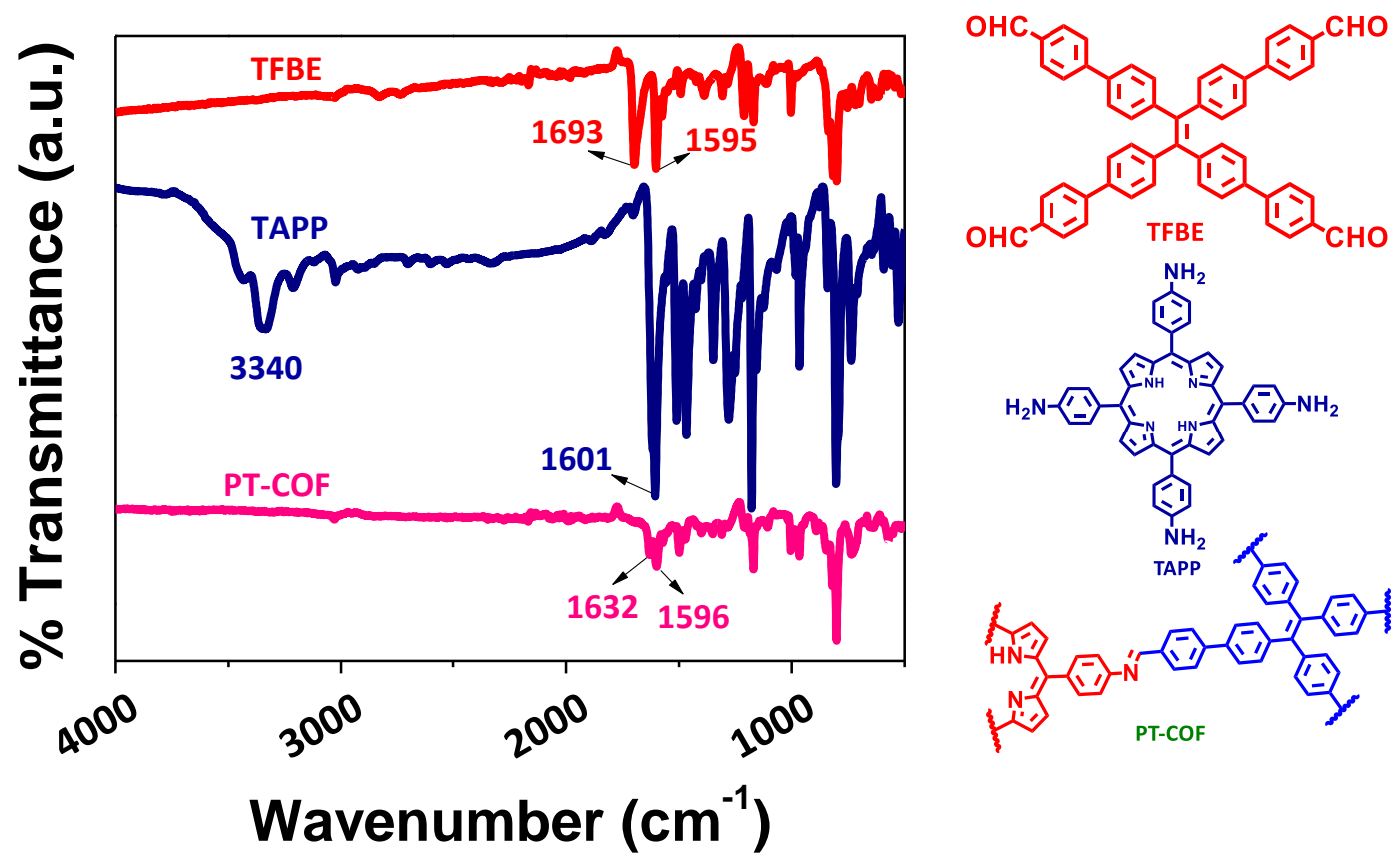

Figure S4: FT-IR spectra of PT-COF (pink), TAPP (navy) and TFBE (red);

S4 Elemental Analysis:

\begin{tabular}{|c|c|c|c|c|}
\hline & & C\% & H\% & N\% \\
\cline { 2 - 5 } PT-COF & Calculated & 84.28 & 4.14 & 11.58 \\
& & & & 11.96 \\
\cline { 2 - 5 } & Experimentally & 83.82 & 4.24 & \\
\hline
\end{tabular}


S5. BET plot calculated from the $\mathbf{N}_{2}$ adsorption data:

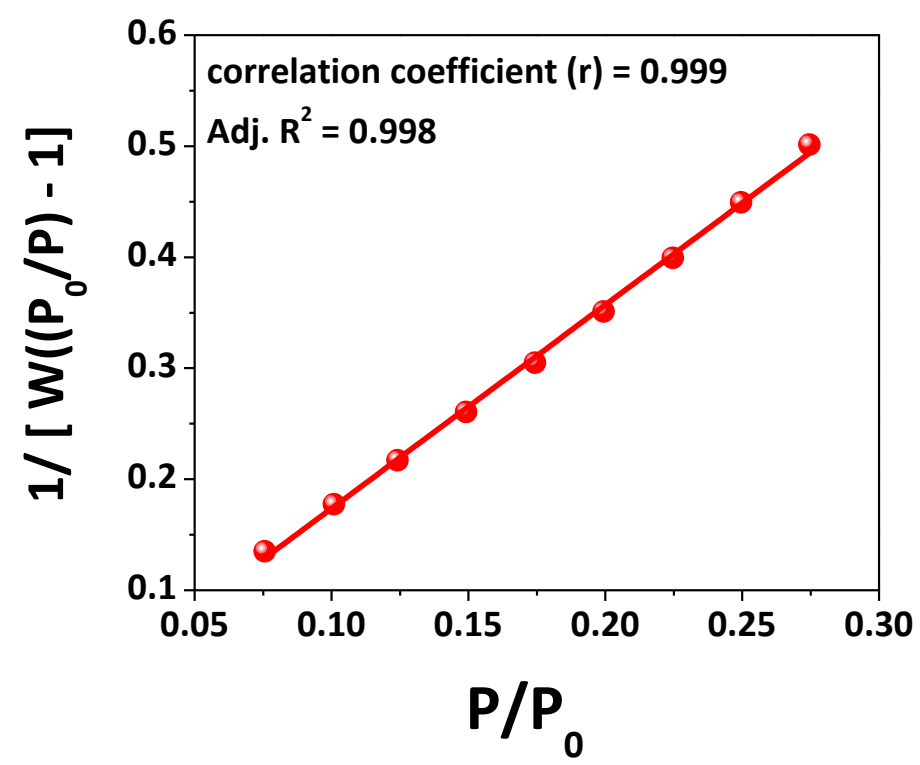

Figure S5: BET plot of the PT-COF from $\mathrm{N}_{2}$ adsorption data. 

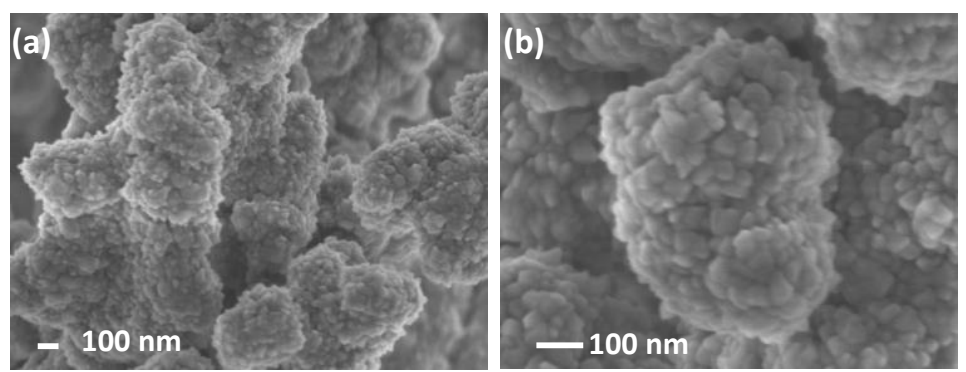

$$
{ }^{12000} \text { (d) }
$$
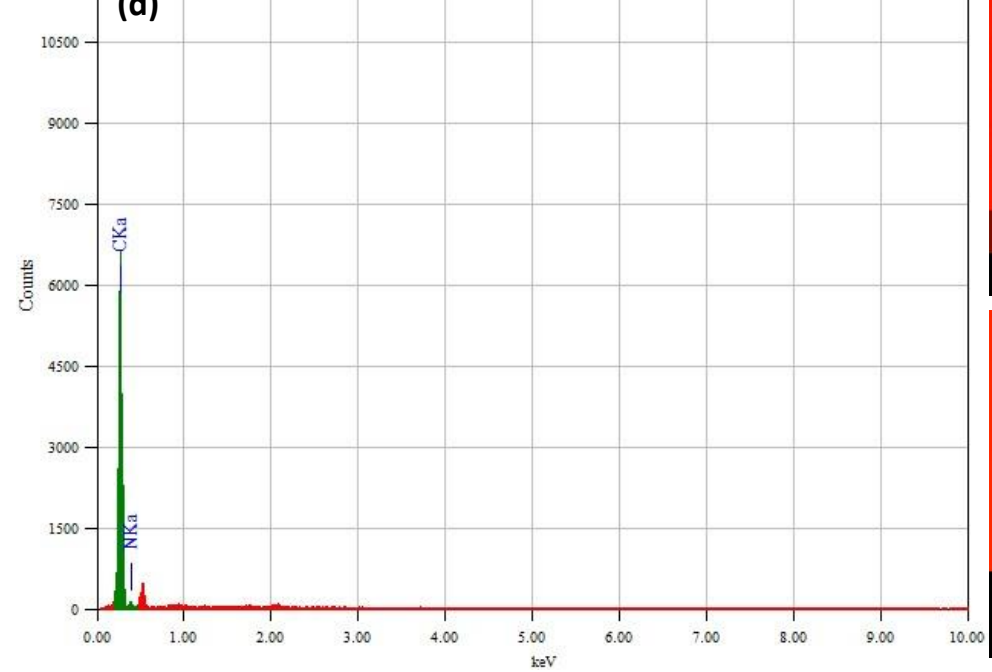

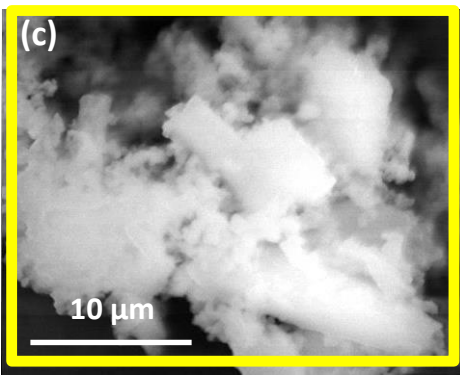

(e)

$10 \mu \mathrm{m}$

(f)

94 Figure S6: (a, b) FE-SEM image of PT-COF, (c) selected area (yellow border) for EDS

95 mapping, (d) EDS analysis distribution of ' $\mathrm{C}$ ' and ' $\mathrm{N}$ ' throughout the PT-COF network. (e)

96 'C' - mapping and (f) ' $\mathrm{N}$ ' - mapping images obtained from EDS mapping. 

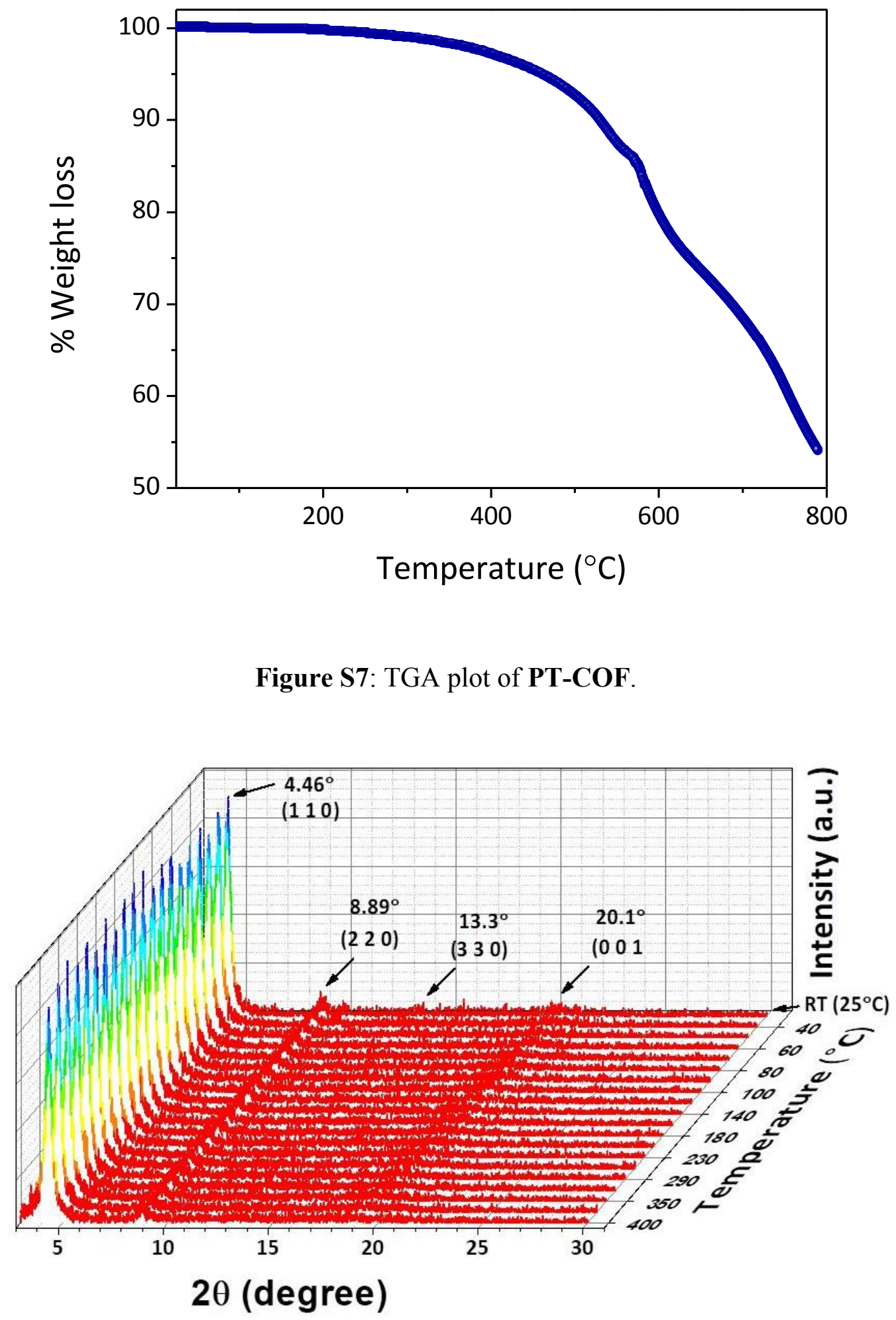

101

Figure S8: Temperature dependent PXRD of PT-COF. 
S8. UV Visible and Photoluminescence study:
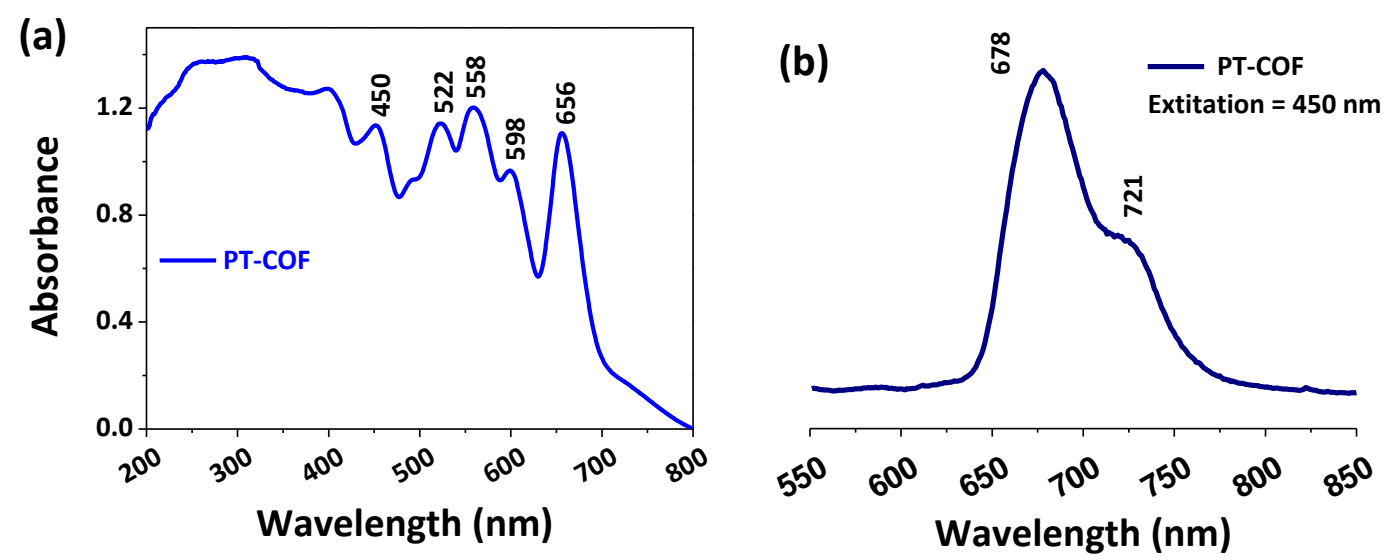

(c)

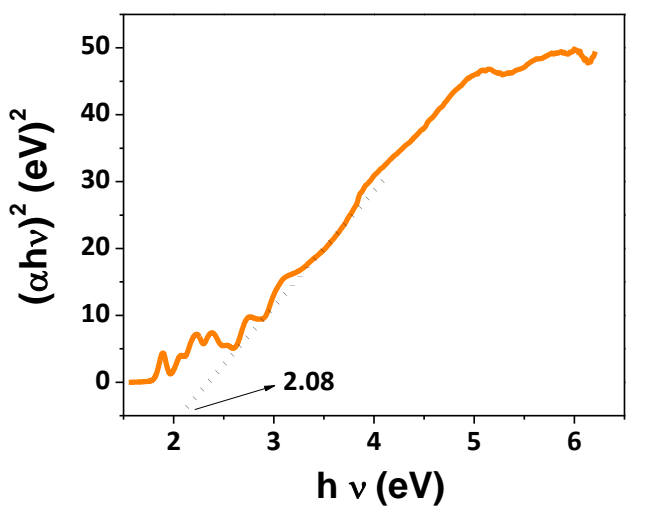

(d)
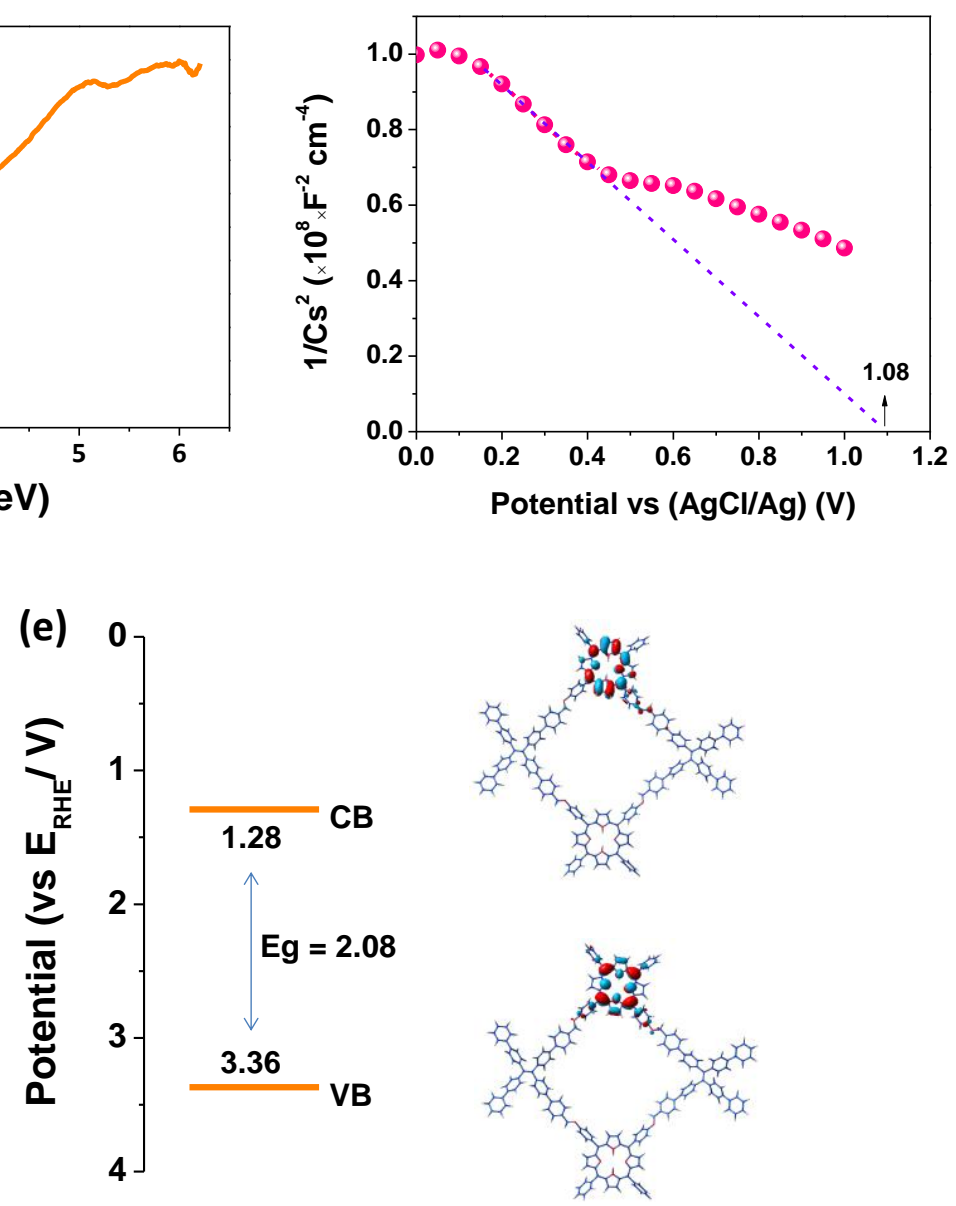

Figure S9: (a) Solid state UV-vis spectrum, (b) PL spectrum and (c) Tauc plot of PT-COF.

(d) Mott-Schottky plot of the PT-COF tested at $1000 \mathrm{~Hz}$ frequencies. Then the conduction bands were determined by the intersections of tangent lines and $\mathrm{X}$ axis. (e) The corresponding band alignment obtained from the Mott-Schottky measurement. 
110 Conductivity measurement of the PT-COF was carried out by making pellet, using four point

111 linear probes with a space $(\mathbf{s})$ between the tips of $2.0 \mathrm{~mm}$. The thickness $(\mathrm{T})$ of the pellet was

$1120.51 \mathrm{~mm}$. The intrinsic bulk electrical conductivity was determined using the equation,

$$
\sigma=\frac{\ln 2}{\pi T} \times \frac{I}{V}
$$

114 where, $\sigma=$ conductivity $\left(\mathrm{S} \mathrm{cm}^{-1}\right), \mathrm{T}=$ thickness of pellet $(\mathrm{cm}), \mathrm{I}=$ measured current $(\mathrm{A}), \mathrm{V}=$ 115 voltage (V).
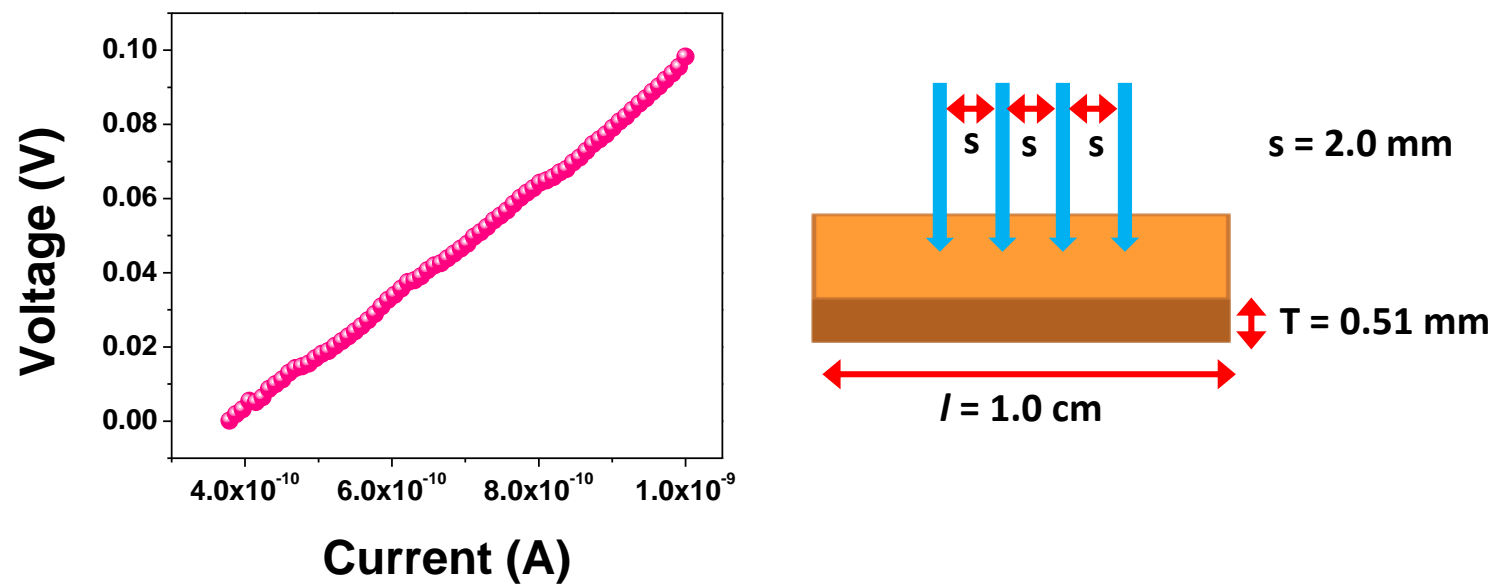

118 Figure S10: I-V plot of the PT-COF for determination of intrinsic conductivity using four point probes. 


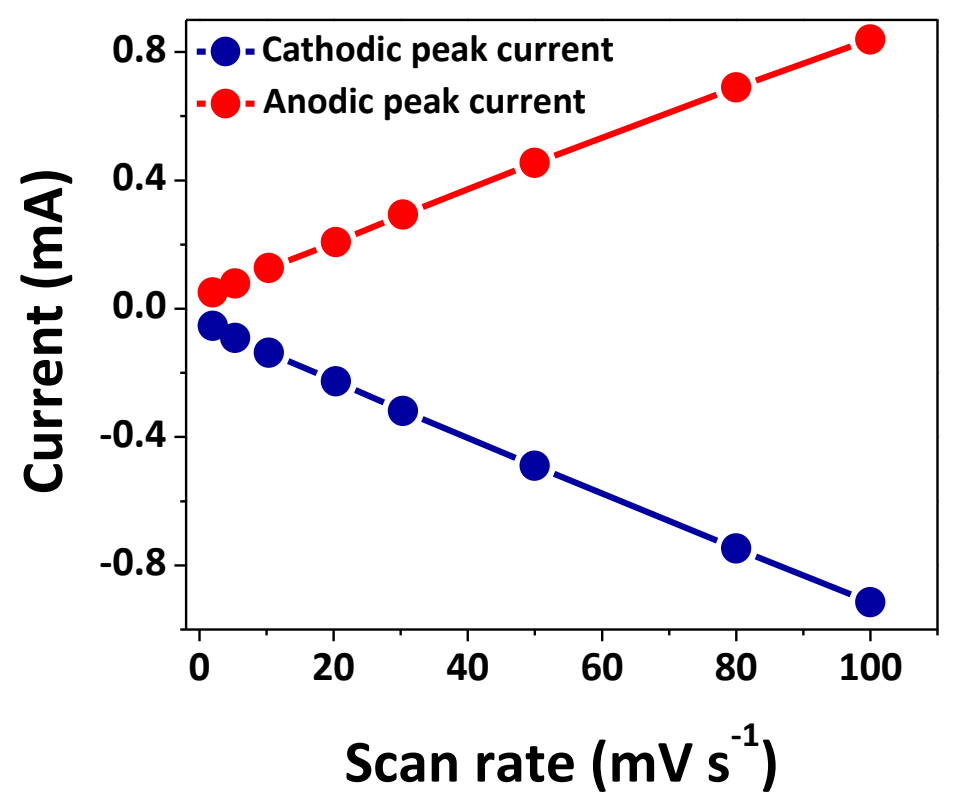

124 Figure S11: (a) Peak current $(\mathrm{mA})$ vs Scan rate $\left(\mathrm{mV} \mathrm{s}^{-1}\right)$ plot obtained from the CV graph, 125 shows the linear relationship, indicates excellent reversible electrochemical process with fast charge transport behaviour which causes very good pseudocapacitive nature of the COF.

127

S10.2 Electrochemical performance of precursors and their physical mixtures:

To check superior electrochemical performance of the PT-COF, we have studied the cyclic voltammetry (CV) and galvanostatic charge-discharge (GCD) analysis of the precursor

131 TFBE aldehyde, TAPP amine and physical mixture of the (TFBE+TAPP). From the GCD profile, the corresponding specific capacitance was calculated (Table S2). 
(a)

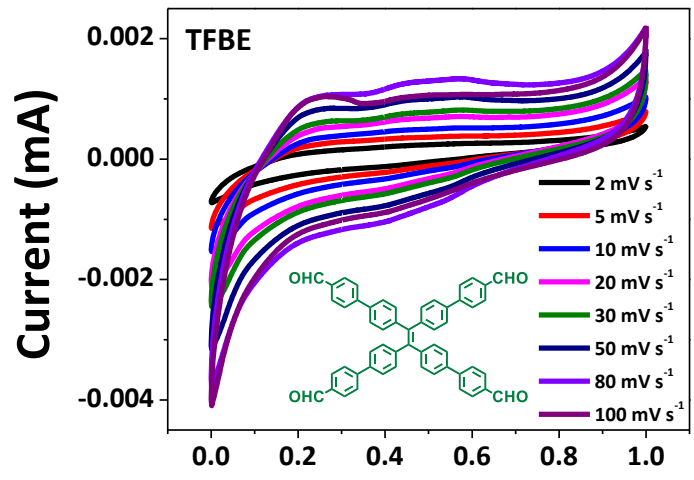

Potential (V) vs $\mathrm{Ag} / \mathrm{AgCl}$

(c)

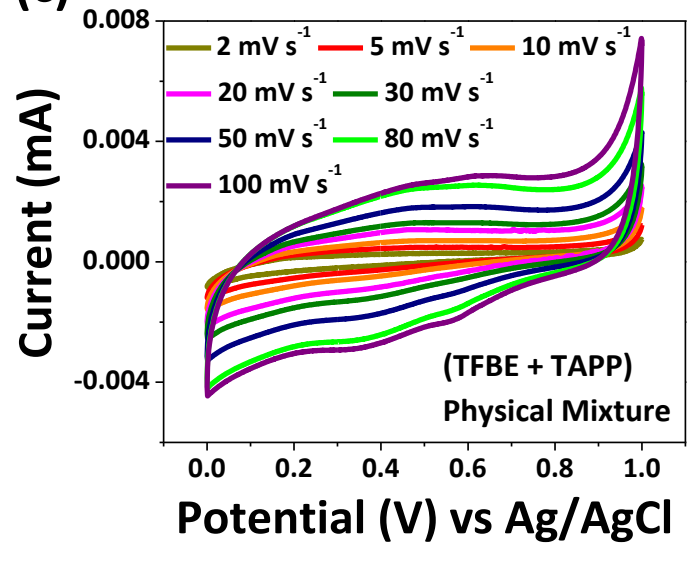

(b)

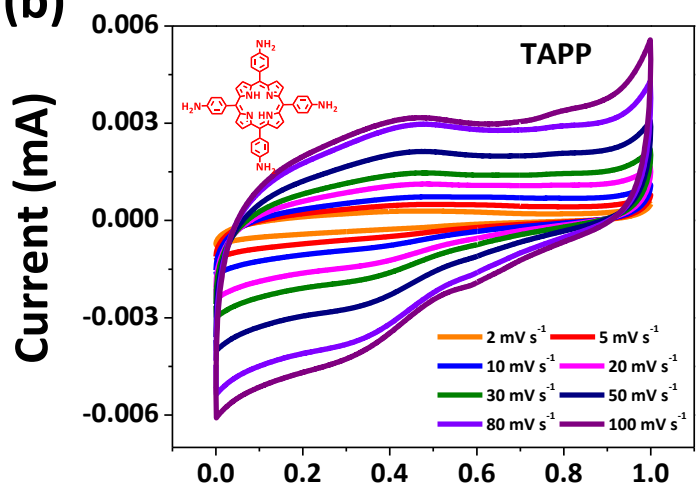

Potential (V) vs $\mathrm{Ag} / \mathrm{AgCl}$

(d)

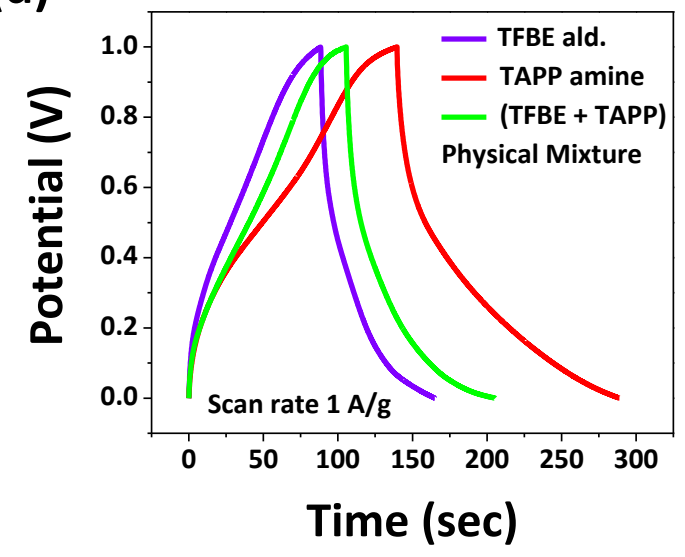

Figure S12: (a) CV graph of TFBE, (b) TAPP and (c) (TFBE+TAPP) physical mixtures at different scan rates. (d) GCD profile of the corresponding material at $1 \mathrm{~A} \mathrm{~g}^{-1}$ current density.

Table S2: Experimental specific capacitance of the precursors (TFBE, TAPP) and their physical mixture:

\begin{tabular}{|c|c|}
\hline Sample Entry & Specific capacitance at $1 \mathrm{~A} \mathrm{~g}^{-1}$ current density \\
\hline TFBE & $78 \mathrm{~F} \mathrm{~g}^{-1}$ \\
\hline TAPP & $150 \mathrm{~F} \mathrm{~g}^{-1}$ \\
\hline (TFBE + TAPP) physical mixture & $102 \mathrm{~F} \mathrm{~g}^{-1}$ \\
\hline PT-COF & $\mathbf{1 4 4 3 ~ F ~ g ~}$ \\
\hline
\end{tabular}


Table S3: Comparison table of specific capacitance of the PT-COF with other literature reported COFs.

\begin{tabular}{|c|c|c|c|c|}
\hline COFs & $\begin{array}{l}\text { Specific capacitance at } \\
\text { (current density) }\end{array}$ & Electrolyte & $\begin{array}{c}\text { Operating } \\
\text { potential window }\end{array}$ & References \\
\hline PT-COF & $1443 \mathrm{~F} \mathrm{~g} \mathrm{~g}^{-1}$ at $\left(1 \mathrm{~A} \mathrm{~g}^{-1}\right)$ & $0.5 \mathrm{M} \mathrm{H}_{2} \mathrm{SO}_{4}$ & $\begin{array}{c}\text { O V - 1 V vs } \\
\text { Ag/AgCl }\end{array}$ & This work \\
\hline Hex-Aza & $663 \mathrm{~F} \mathrm{~g}^{-1}$ at $\left(1 \mathrm{~A} \mathrm{~g}^{-1}\right)$ & $1.0 \mathrm{M} \mathrm{H}_{2} \mathrm{SO}_{4}$ & $\begin{array}{c}-1.1 \mathrm{~V}-0.1 \mathrm{~V} \text { vs } \\
\mathrm{Hg}-\mathrm{Hg}_{2} \mathrm{SO}_{4}\end{array}$ & $\begin{array}{c}\text { Avd. Energy } \\
\text { Mater. 2020, } 10, \\
2001673- \\
2001681 .\end{array}$ \\
\hline $\begin{array}{l}\text { TpOMe- } \\
\text { DAQ }\end{array}$ & $\begin{array}{c}135 \mathrm{~F} \mathrm{~g}^{-1} \text { at }\left(0.35 \mathrm{~A} \mathrm{~g}^{-}\right. \\
\left.{ }^{-}\right)\end{array}$ & $2.0 \mathrm{M} \mathrm{H}_{2} \mathrm{SO}_{4}$ & $\begin{array}{c}-0.5 \mathrm{~V}-0.5 \mathrm{~V} \text { vs } \\
\mathrm{Hg}-\mathrm{Hg}_{2} \mathrm{SO}_{4}\end{array}$ & $\begin{array}{c}\text { J. Am. Chem. } \\
\text { Soc. } \\
\text { 2018, 140, } \\
10941-10945 .\end{array}$ \\
\hline DAAQ-TFP & $\begin{array}{c}48 \pm \underset{\left.\mathrm{mV} \mathrm{s}^{-1}\right)}{10 \mathrm{~F} \mathrm{~g}^{-1}} \text { at }(10 \\
\text { (1) }\end{array}$ & $1.0 \mathrm{M} \mathrm{H}_{2} \mathrm{SO}_{4}$ & $\begin{array}{l}-0.3 \mathrm{~V} \text { to } 0.3 \mathrm{~V} \\
\text { (vs } \mathrm{Ag} / \mathrm{AgCl} \text { ) }\end{array}$ & $\begin{array}{c}\text { J. Am. Chem. } \\
\text { Soc. 2013, } 135 \\
16821-16824 .\end{array}$ \\
\hline TTF-COF1 & $752 \mathrm{~F} \mathrm{~g}^{-1}$ at $\left(1 \mathrm{~A} \mathrm{~g}^{-1}\right)$ & $3 \mathrm{M} \mathrm{KOH}$ & $\begin{array}{c}0 \mathrm{~V} \text { to } 0.5 \mathrm{~V} \text { (vs } \\
\mathrm{Hg} / \mathrm{HgO})\end{array}$ & $\begin{array}{c}\text { Chem. Commun. } \\
\mathbf{2 0 2 0}, 56, \\
14187-14190 .\end{array}$ \\
\hline PDA-MA & $335 \mathrm{~F} \mathrm{~g}^{-1}$ at $\left(1.0 \mathrm{~A} \mathrm{~g}^{-1}\right)$ & $6 \mathrm{M} \mathrm{KOH}$ & $0 \mathrm{~V}$ to $1.5 \mathrm{~V}$ & $\begin{array}{c}\text { ACS Appl. } \\
\text { Mater. } \\
\text { Interfaces 2019, } \\
11,26355- \\
26363 .\end{array}$ \\
\hline $\begin{array}{l}\text { TpPa- }(\mathrm{OH})_{2-}- \\
\text { COF }\end{array}$ & $344 \mathrm{~F} \mathrm{~g}^{-1}$ at $\left(1 \mathrm{~A} \mathrm{~g}^{-1}\right)$ & $\begin{array}{c}1.0 \mathrm{M} \\
\text { Phosphate } \\
\text { buffer } \\
(\mathrm{pH}=7.2)\end{array}$ & $\begin{array}{c}-0.2 \mathrm{~V} \text { to } 0.5 \mathrm{~V} \\
\text { (vs SCE) }\end{array}$ & $\begin{array}{c}\text { Chem. Mater. } \\
\text { 2017, 29, 2074- } \\
2080 .\end{array}$ \\
\hline Dq1Da1Tp & $\begin{array}{c}111 \mathrm{~F} \mathrm{~g}^{-1}(1.56 \mathrm{~mA} \\
\left.\mathrm{cm}^{-2}\right)\end{array}$ & $1.0 \mathrm{M} \mathrm{H}_{2} \mathrm{SO}_{4}$ & $\begin{array}{l}-0.5 \mathrm{~V} \text { to } 0.3 \mathrm{~V} \\
\left(\mathrm{vs} \mathrm{Hg}-\mathrm{Hg}_{2} \mathrm{SO}_{4}\right)\end{array}$ & $\begin{array}{c}\text { ACS Appl. } \\
\text { Mater. } \\
\text { Interfaces, } \mathbf{2 0 1 8}, \\
\text { 10, 28139- } \\
28146 .\end{array}$ \\
\hline $\begin{array}{l}\text { TPPDA- } \\
\text { TPTPE }\end{array}$ & $237.1 \mathrm{~F} \mathrm{~g}^{-1}$ at $\left(2 \mathrm{~A} \mathrm{~g}^{-1}\right)$ & $1.0 \mathrm{M} \mathrm{KOH}$ & $\begin{array}{c}-0.92 \mathrm{~V} \text { to }+0.18 \\
\mathrm{~V}(\mathrm{Hg} / \mathrm{HgO})\end{array}$ & $\begin{array}{c}\text { Chem. Commun. } \\
\mathbf{2 0 1 9}, 55, \\
14890-14893\end{array}$ \\
\hline Ni-MOF-24 & $\begin{array}{c}1127 \mathrm{~F} \mathrm{~g}^{-1} \text { at }\left(0.5 \mathrm{~A} \mathrm{~g}^{-}\right. \\
1)\end{array}$ & $\begin{array}{c}6.0 \\
\mathrm{M} \mathrm{KOH}\end{array}$ & $\begin{array}{l}0.0 \mathrm{~V} \text { to }+0.35 \mathrm{~V} \\
\text { (vs SCE) }\end{array}$ & $\begin{array}{c}\text { J. Mater. Chem. } \\
\text { A 2014, 2, } \\
\text { 16640-16644. }\end{array}$ \\
\hline Ni Co-MOF & $\begin{array}{c}1202 \mathrm{~F} \mathrm{~g}^{-1} \text { at }\left(1.0 \mathrm{~A} \mathrm{~g}^{-}\right. \\
1)\end{array}$ & $2.0 \mathrm{M} \mathrm{KOH}$ & $\begin{array}{l}0.0 \mathrm{~V} \text { to }+0.6 \mathrm{~V} \\
\text { (vs SCE) }\end{array}$ & $\begin{array}{c}\text { ACS Appl. } \\
\text { Energy Mater. } \\
\text { 2019, 2, 2063- } \\
2071 .\end{array}$ \\
\hline 65Ni-MDH & $875 \mathrm{C} \mathrm{g}^{-1}$ at $\left(1.0 \mathrm{~A} \mathrm{~g}^{-1}\right)$ & - & $\begin{array}{l}0.0 \mathrm{~V} \text { to }+0.5 \mathrm{~V} \\
(\mathrm{vs} \mathrm{Ag} / \mathrm{AgCl})\end{array}$ & $\begin{array}{c}\text { ACS Energy } \\
\text { Lett. 2017, } 2 \text {, } \\
1263-1269 .\end{array}$ \\
\hline
\end{tabular}




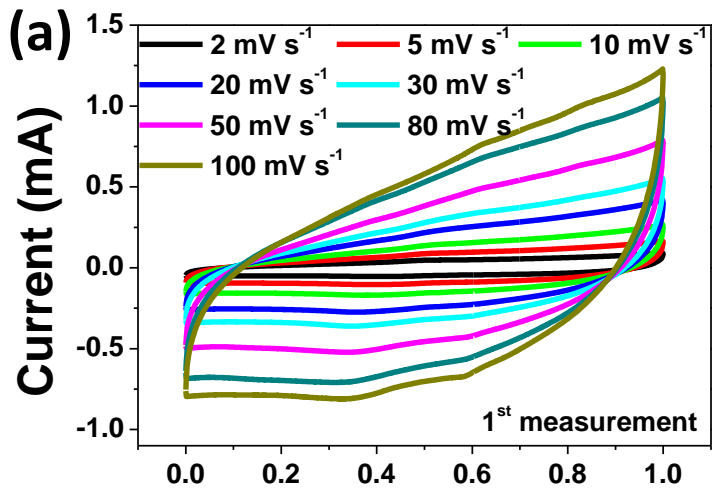

Potential (V) vs $\mathrm{Ag} / \mathrm{AgCl}$

(c)

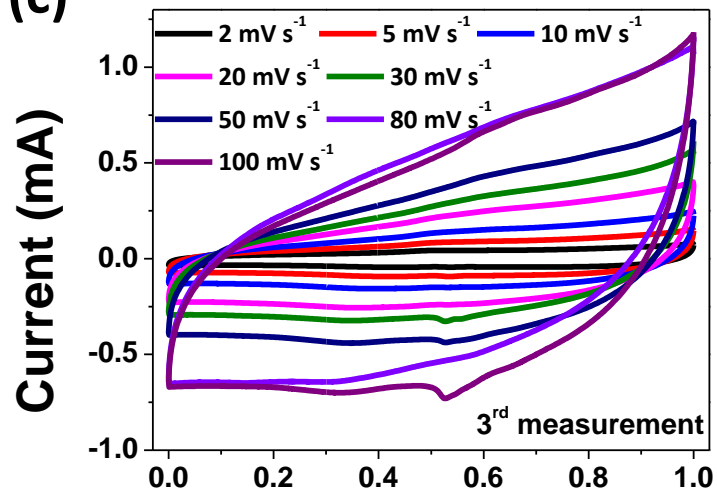

Potential (V) vs $\mathrm{Ag} / \mathrm{AgCl}$

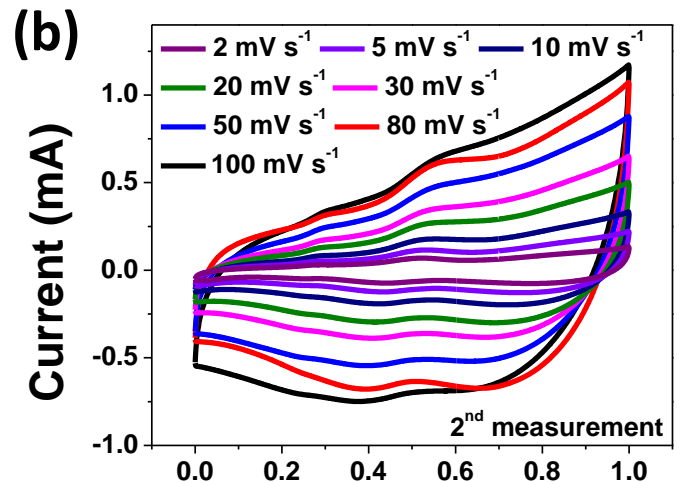

(d)

Potential (V) vs Ag/AgCl

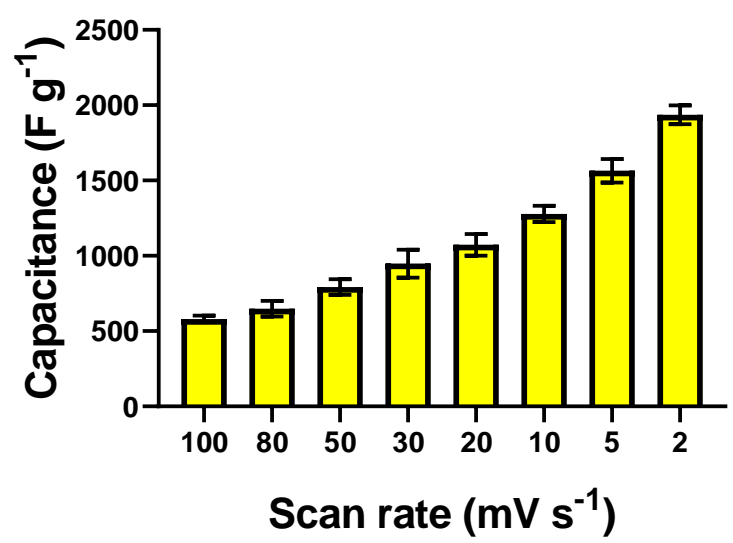

Figure S13: Three independent CV measurements (a, b and c) of the PT-COF and (d) Error bars were obtained from the three $\mathrm{CV}$ measurements.

Table S4: Specific capacitance values of the PT-COF at three repeat measurements obtained by $\mathrm{CV}$ analysis:

\begin{tabular}{|c|c|c|c|}
\hline $\begin{array}{c}\text { Scan rate } \\
\left(\mathrm{mV} \mathrm{s}^{-1}\right)\end{array}$ & $\begin{array}{c}\text { Specific Capacitance }\left(\mathrm{F} \mathrm{g}^{-1}\right) \\
\text { in } 1^{\text {st }} \text { batch }\end{array}$ & $\begin{array}{c}\text { Specific Capacitance }(\mathrm{F} \\
\left.\mathrm{g}^{-1}\right) \text { in } 2^{\text {nd }} \text { batch }\end{array}$ & $\begin{array}{c}\text { Specific Capacitance } \\
\left(\mathrm{F} \mathrm{g}^{-1}\right) \text { in } 3^{\text {rd }} \text { batch }\end{array}$ \\
\hline $\mathbf{1 0 0}$ & 550.90498 & 558.1567 & 583 \\
\hline $\mathbf{8 0}$ & 658 & 593.6367 & 698 \\
\hline $\mathbf{5 0}$ & 841.90476 & 752.7533 & 852 \\
\hline $\mathbf{3 0}$ & 1048.63582 & 883.0433 & 1055 \\
\hline $\mathbf{2 0}$ & 1244.63217 & 1022.6 & 1156 \\
\hline $\mathbf{1 0}$ & 1473.33333 & 1232.09 & 1337 \\
\hline $\mathbf{5}$ & 1632.50231 & 1503.56 & 1653 \\
\hline $\mathbf{2}$ & 1875 & 1998 & 1938 \\
\hline
\end{tabular}



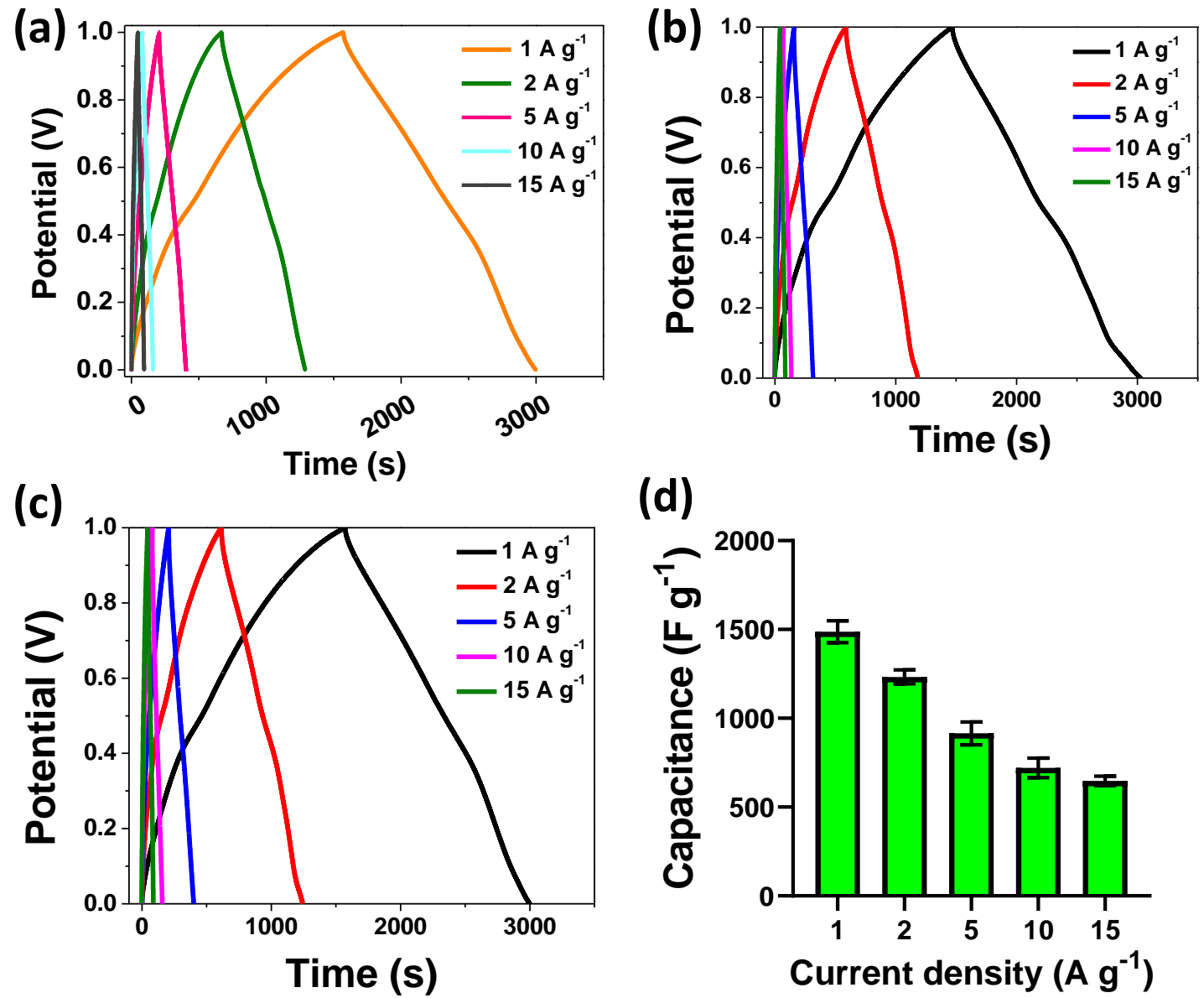

(d)

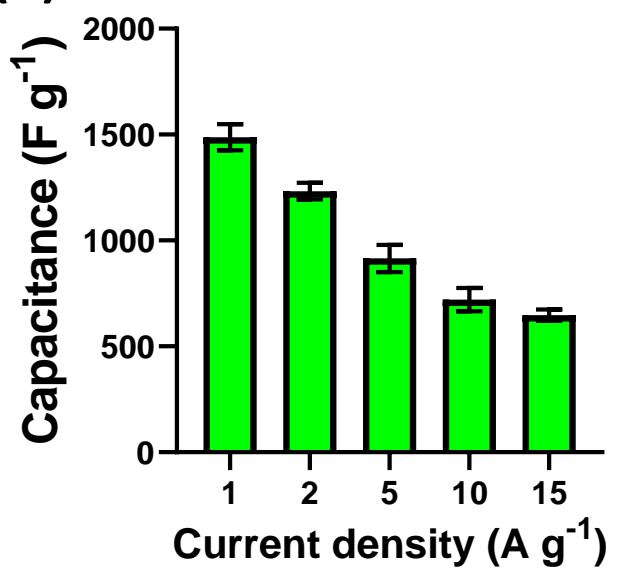

Figure S14: GCD graph at three repeate measurement $(a, b, c)$ and the corresponding error

153 bar graph of the capacitance value with respect to current density (d).

Table S5: Specific capacitance values for three repeat measurement of the PT-COF obtained by GCD analysis.

\begin{tabular}{|c|c|c|c|}
\hline $\begin{array}{c}\text { Current } \\
\text { density }\left(\mathrm{A} \mathrm{g}^{-}\right. \\
1\end{array}$ & $\begin{array}{c}1^{\text {st }} \text { Batch Specific } \\
\text { Capacitance }\left(\mathrm{F} \mathrm{g}^{-1}\right)\end{array}$ & $\begin{array}{c}2^{\text {nd }} \text { Batch Specific } \\
\text { Capacitance }\left(\mathrm{F} \mathrm{g}^{-1}\right)\end{array}$ & $\begin{array}{c}3^{\text {rd }} \text { batch Specific } \\
\text { Capacitance }\left(\mathrm{F} \mathrm{g}^{-1}\right)\end{array}$ \\
\hline $\mathbf{1}$ & 1443 & 1550 & 1486 \\
\hline $\mathbf{2}$ & 1262 & 1188 & 1248 \\
\hline $\mathbf{5}$ & 985 & 858 & 902 \\
\hline $\mathbf{1 0}$ & 780 & 670 & 712 \\
\hline $\mathbf{1 5}$ & 675 & 645 & 622 \\
\hline
\end{tabular}


Further, we have performed the cyclic voltammetry (CV) measurement in neutral medium $\left(1 \mathrm{M} \mathrm{Na}_{2} \mathrm{SO}_{4}\right)$ to speculate the pseudo capacitance contribution of the PT-COF in acidic media (Figure S15). From the CV graph, it is observed that there is no faradaic peak of the PT-COF in neutral medium. This confirms that the PT-COF shows only EDL capacitance in neutral medium. It is observed that the PT-COF a capacitance value of $1390 \mathrm{~F} \mathrm{~g}^{-1}$ at $2 \mathrm{mV} \mathrm{s}^{-1}$ scan rate in $1 \mathrm{M} \mathrm{N}_{2} \mathrm{SO}_{4}$ (Table S6)

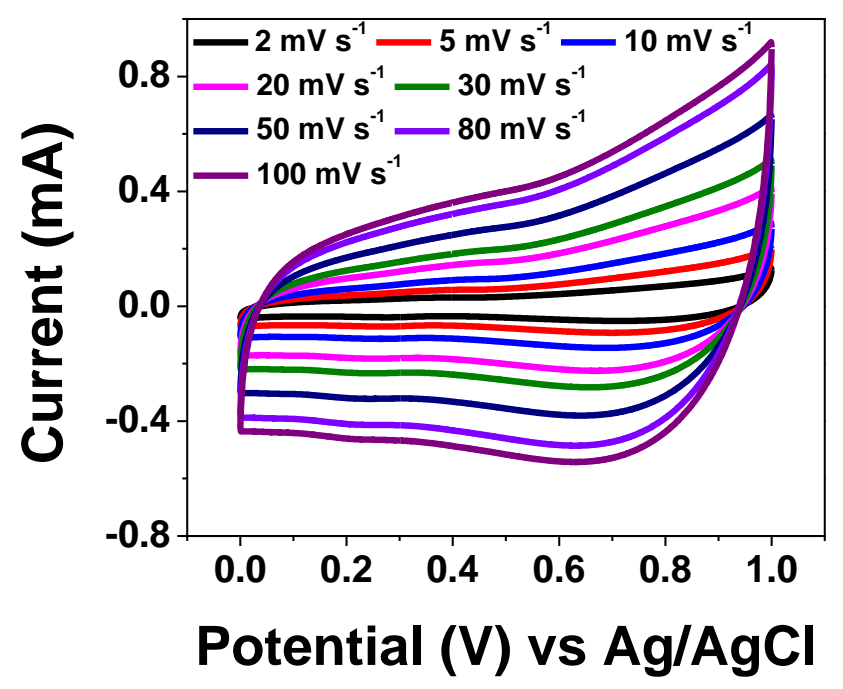

Figure S15: Cyclic voltammetry data of the PT-COF in $1 \mathrm{M} \mathrm{Na}_{2} \mathrm{SO}_{4}$.

Table S6: Specific capacitance values of PT-COF at $\mathrm{H}_{2} \mathrm{SO}_{4}$ and $\mathrm{Na}_{2} \mathrm{SO}_{4}$ at different scan rates.

\begin{tabular}{|c|c|c|}
\hline Scan rate $\left(\mathrm{mV} \mathrm{s}^{-1}\right)$ & $\begin{array}{c}\text { Specific Capacitance in } \mathrm{H}_{2} \mathrm{SO}_{4} \\
\left(\mathrm{~F} \mathrm{~g} \mathrm{~g}^{-1}\right)\end{array}$ & $\begin{array}{c}\text { Specific Capacitance in } \mathrm{Na}_{2} \mathrm{SO}_{4} \\
\left(\mathrm{~F} \mathrm{~g}^{-1}\right)\end{array}$ \\
\hline 2 & 1875 & 1390 \\
\hline 5 & 1632 & 990 \\
\hline 10 & 1473 & 773 \\
\hline 20 & 1244 & 600 \\
\hline 30 & 1048 & 505 \\
\hline 50 & 841 & 410 \\
\hline 80 & 658 & 328 \\
\hline 100 & 550 & 293 \\
\hline
\end{tabular}




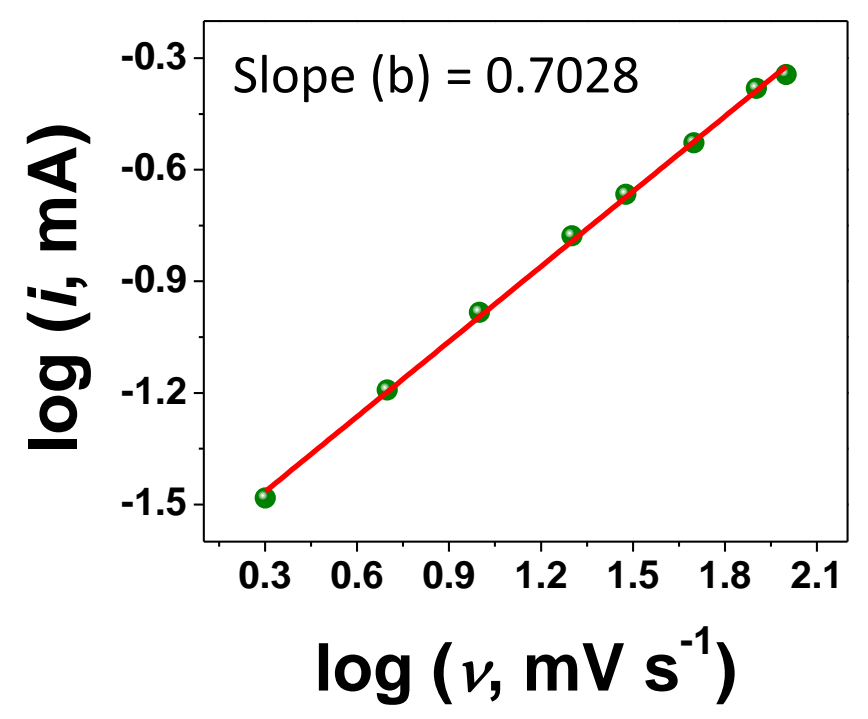

174 Figure S16: $\log (i)$ vs $\log (v)$ plot at $0.4 \mathrm{~V}$ (anodic) where the peak current density as a function of scan rate and the slope gives the ' $b$ ' value.

S10.4 Contribution of EDL capacitance $\left(C_{E D L}\right)$ and pseudocapacitance $\left(C_{p}\right)$ :

S10.4.1 Estimation of maximum capacitance $\left(C_{T}\right)$ by Trasatti method:

A linear correlation between the reciprocal of the calculated gravimetric capacitance $\left(\mathrm{C}^{-1}\right) \mathrm{vs}$ the square root of scan rates $\left(v^{1 / 2}\right)$ follows the equation (assuming that that ions unrestrictedly diffuse to electrode/electrolyte interface from bulk electrolyte),

$$
\mathrm{C}^{-1}=\operatorname{Constant}(v)^{1 / 2}+\mathrm{C}_{\mathrm{T}}^{-1}
$$

where, ' $\mathrm{C}_{\mathrm{T}}$ ' is the maximum capacitance which represents the sum of EDL capacitance $\left(\mathrm{C}_{\mathrm{EDL}}\right)$ and pseudo capacitance $\left(\mathrm{C}_{\mathrm{p}}\right)$. The $\mathrm{C}_{\mathrm{T}}$ value can be obtained from the reciprocal of the y-intercept of the $\mathrm{C}^{-1}$ vs $v^{1 / 2}$ plot. 


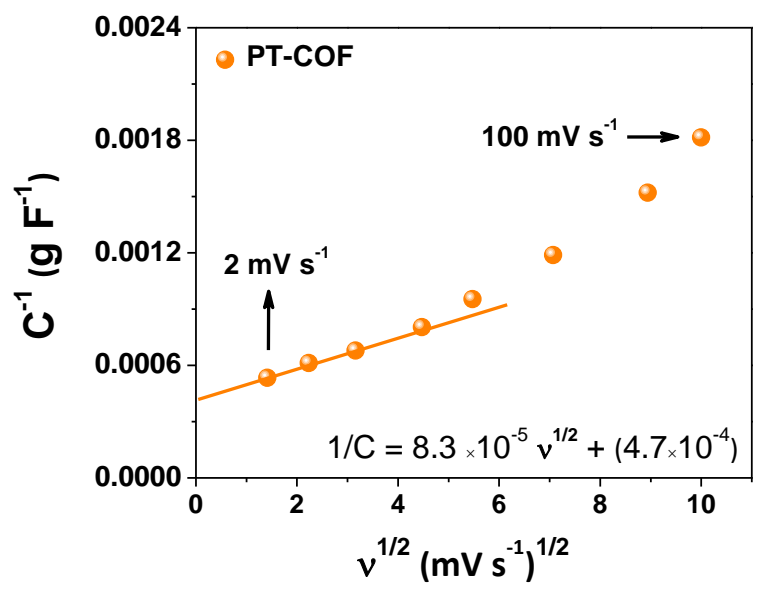

189 Figure S17: $C^{-1}$ vs $v^{1 / 2}$ plot. From the reciprocal of the y-intercept, the $C_{T}$ value can be obtained.

S10.4.2 Evaluation of maximum electrical double layer capacitance (CEDL):

A linear correlation between the experimental gravimetric capacitance (C) vs reciprocal of square root of scan rates would follow the equation (assuming semi-infinite diffusion),

$$
\mathrm{C}=\text { constant }(v)^{-1 / 2}+\mathrm{C}_{\mathrm{EDL}}
$$

where $C, v$ and $C_{E D L}$ is the experimental gravimetric capacitance, scan rate and maximum electrical double layer capacitance, respectively. Taking the linear fit of the plot at low scan rates and extrapolates the fitting line to y-axis gives the $C_{E D L}$.

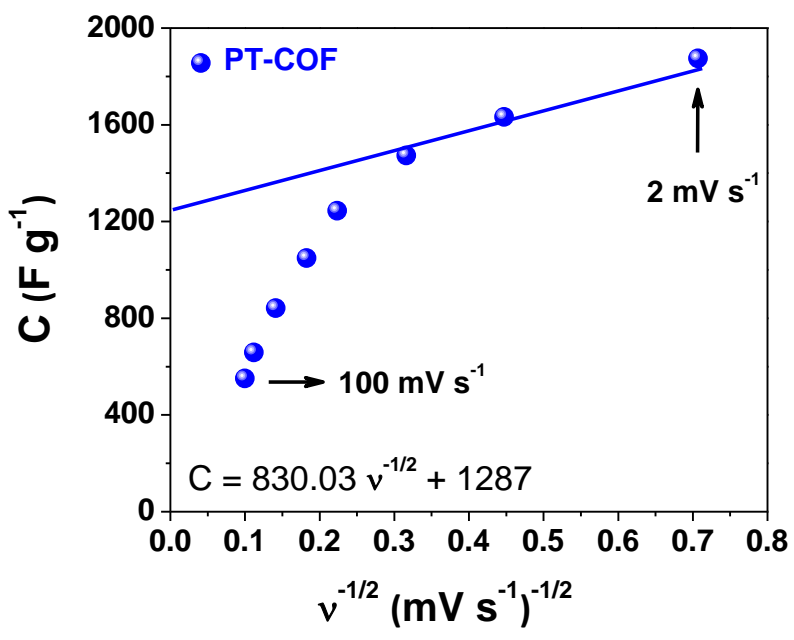

199 Figure S18: C vs $v^{-1 / 2}$ plot. The linear fit of the plot at low scan rates and extrapolates the fitting line to $\mathrm{y}$-axis gives the $C_{E D L}$. 
Subtraction of $C_{E D L}$ from $C_{T}$ yields the maximum pseudo-capacitance $\left(C_{P}\right)$.

203

204

205

206

207

208

209

210

211

212

213

Hence, $C_{p}=\left(C_{T}-C_{E D L}\right)=(2127.6-1287)=840.6$

\section{S10.4.3 Evaluation of the percentage of capacitance contribution:}

$$
\begin{aligned}
& \% C_{E D L}=\frac{C_{E D L}}{C_{T}} \times 100 \%=60.4 \% \\
& \% C_{P}=\frac{C_{P}}{C_{T}} \times 100 \%=39.6 \%
\end{aligned}
$$

From the Dunn analysis, the fraction of each component was calculated using the following equation

$$
\mathrm{i}_{\mathrm{p}}=\mathrm{k}_{1} v+\mathrm{k}_{2} \mathrm{v}^{0.5}
$$

where, $\mathrm{i}_{\mathrm{p}}=$ current at certain potential, ' $\mathrm{k}_{1}$ ' and ' $\mathrm{k}_{2}$ ' are the constant at certain voltage. $60 \%$ $\mathrm{C}_{\mathrm{p}}$ contribution was observed at a scan rate of $5 \mathrm{mV} \mathrm{s}^{-1}$ and there is a gradual decrease of $\mathrm{C}_{\mathrm{p}}$ value with increasing the scan rate. At higher scan rate of $100 \mathrm{mv} \mathrm{s}^{-1}, \mathrm{C}_{\mathrm{EDL}}$ value reaches upto $75 \%$ which indicates the prominent contribution of EDL capacitance.

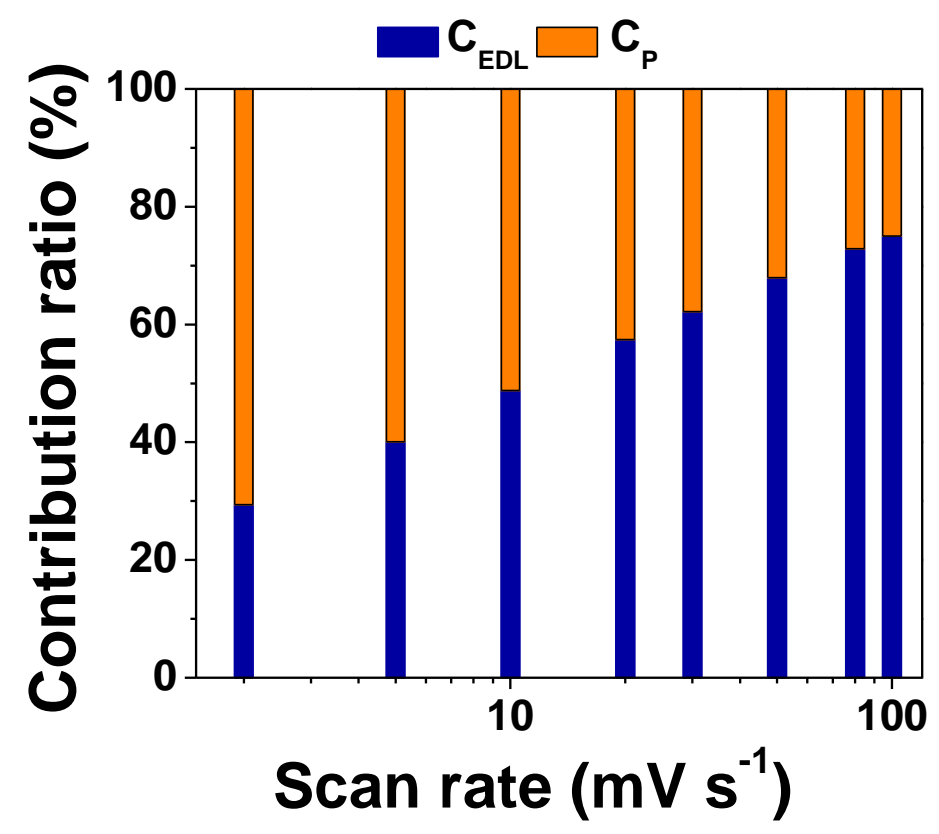

Figure S19: $C_{E D L}$ and $C_{P}$ contribution ratio in bar diagram at different scan rates by Dunn analysis. 

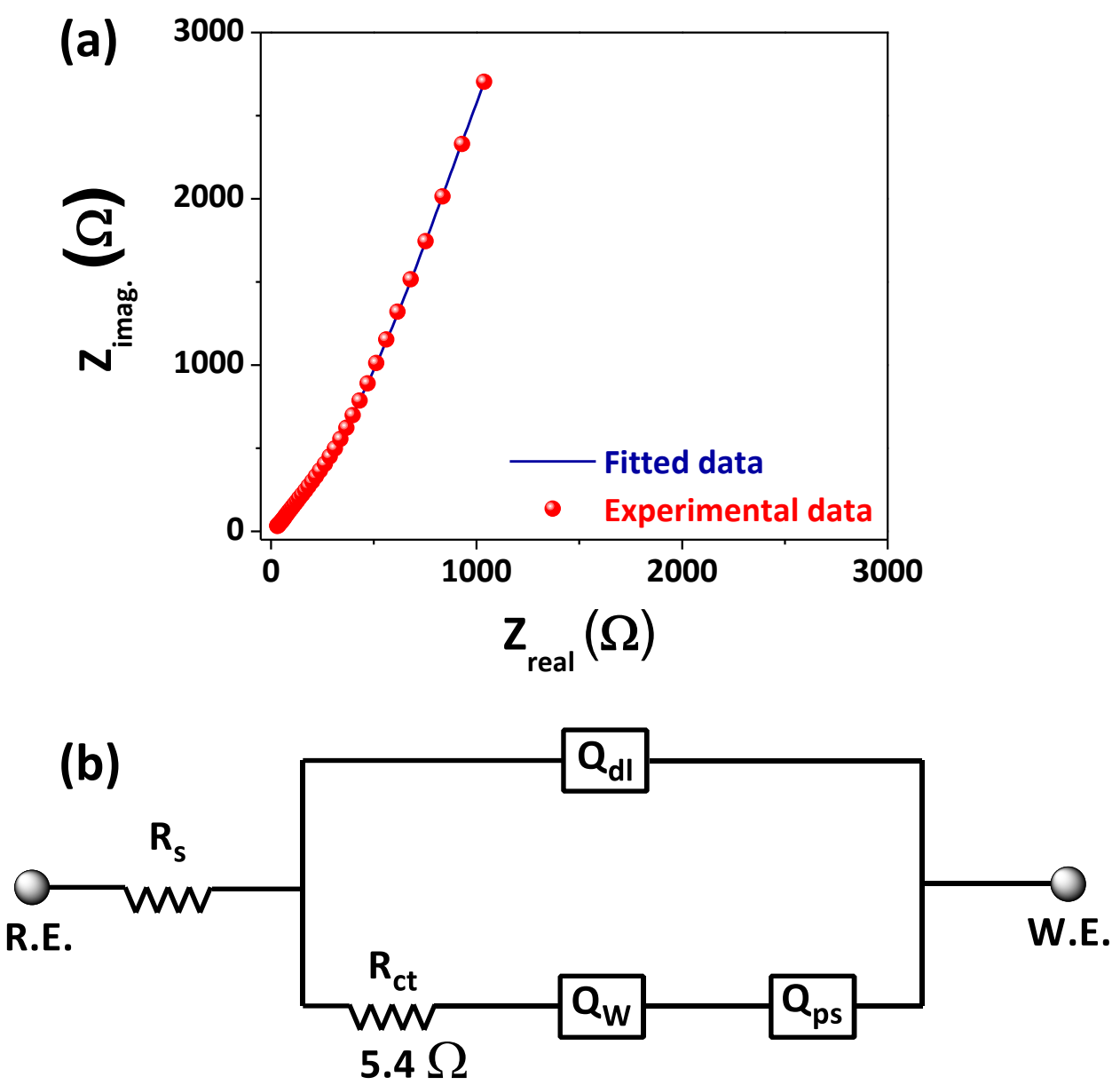

Figure S20: (a) Nyquist plot of PT-COF (red circle) along with best fitted EIS result (solid navy line) using the equivalent circuit. (b) Equivalent circuit of the of the fitted EIS data. In this circuit, $\mathrm{R}_{\mathrm{s}}=$ solution resistance, $\mathrm{R}_{\mathrm{ct}}=$ charge transfer resistance due to electron transfer, $\mathrm{Q}_{\mathrm{dl}}=$ charging capacity in the electrode-electrolyte interface, $\mathrm{Q}_{\mathrm{w}}=$ Warburg diffusion of the proton within the interface, $\mathrm{Q}_{\mathrm{ps}}=$ pseudocapacitive charging at low frequency region. From the fitted data, it was observed that the ' $n$ ' value obtained from the constant phase element $\left(\mathrm{Q}_{\mathrm{dl}}\right)$ for double layer charging, was 0.7 which is lower than the constant phase element $\mathrm{Q}_{\mathrm{ps}}$

227 (0.988). This indicates a pseudocapacitive charging was happening inside the COF 228 framework. ${ }^{2,3}$ 


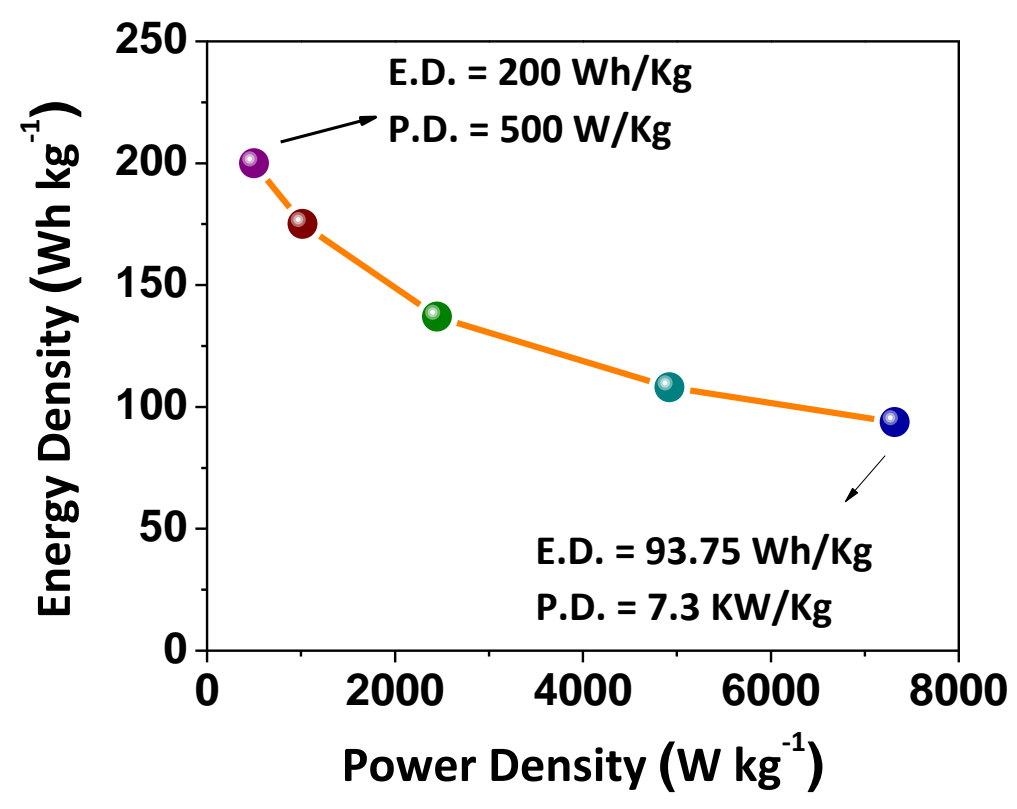

231

232 Figure S21: Ragone plot (energy density vs. power density) of the COF. Highest power 233 density (P.D.) of $7.3 \mathrm{~kW} \mathrm{~kg}^{-1}$ was observed at high scan rate which outperformed the other 234 COF-based materials. At very low scan rate the highest energy density (E.D.) of $200 \mathrm{~W} \mathrm{~h} \mathrm{~kg}^{-1}$ was observed for the PT-COF.

S11. Density of state (DOS) calculation:

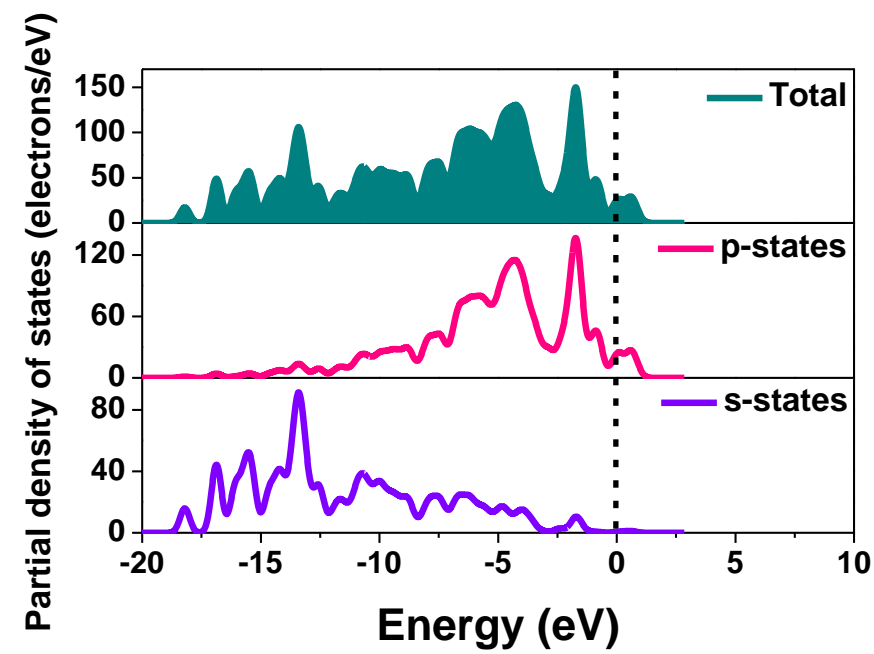

Figure S22: DOS calculation of the PT-COF to determine the electronic states near Fermi region. 
S12. Post cycle characterization of the PT-COF:

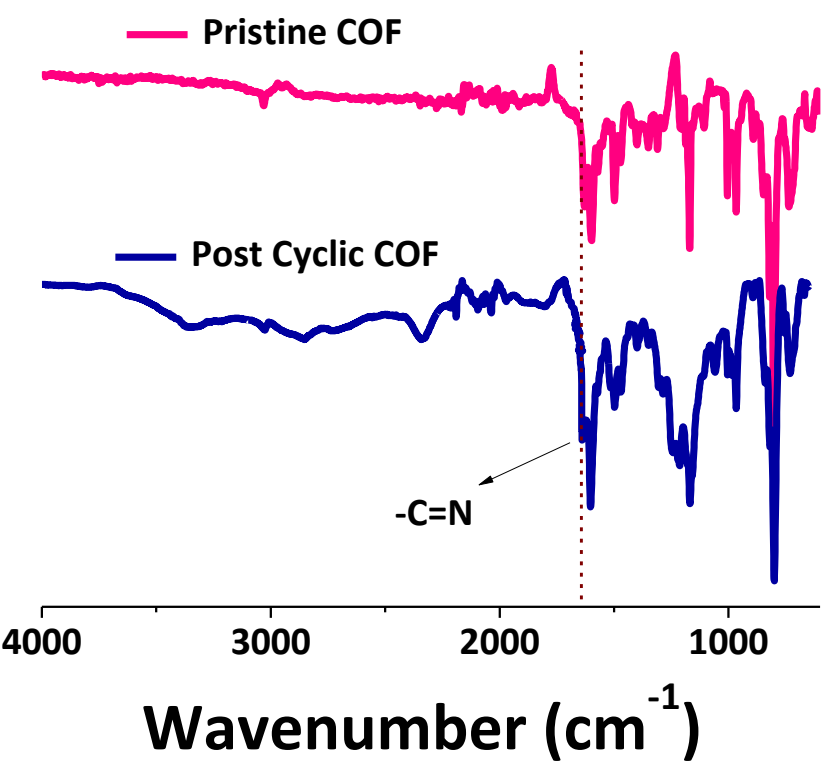

Figure S23: Post cycle IR graph of the PT-COF is compared with pristine COF.

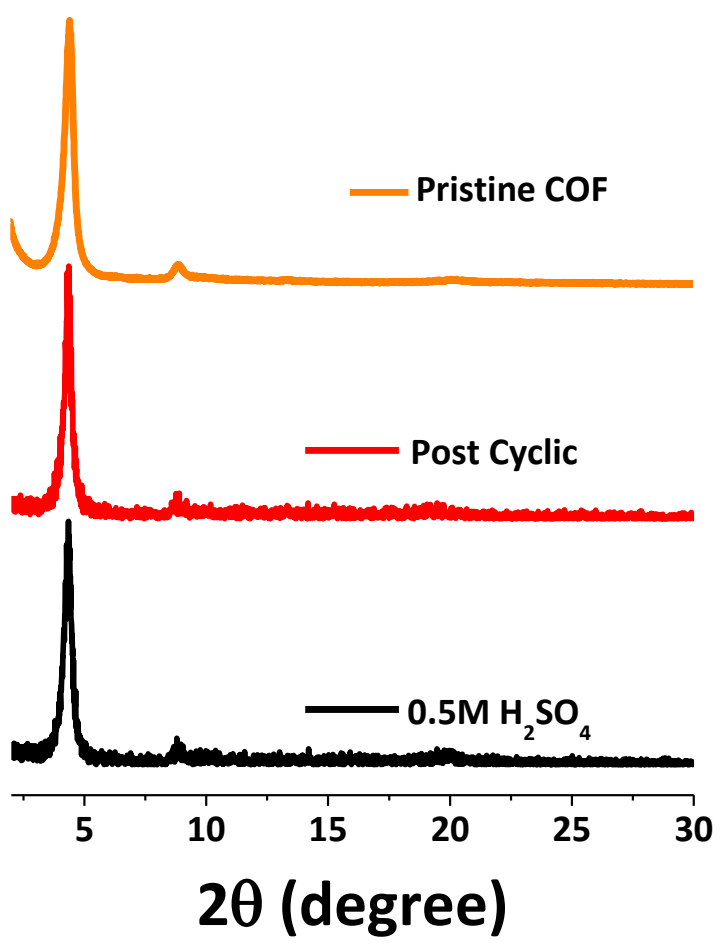

244 Figure S24: P-XRD pattern of PT-COF after post cycle (red line color) is compared with the 245 pristine COF (orange line). PXRD pattern of the COF after recover the sample from $0.5 \mathrm{M}$ $246 \mathrm{H}_{2} \mathrm{SO}_{4}$ (immersed for 4 days) (black line color). 

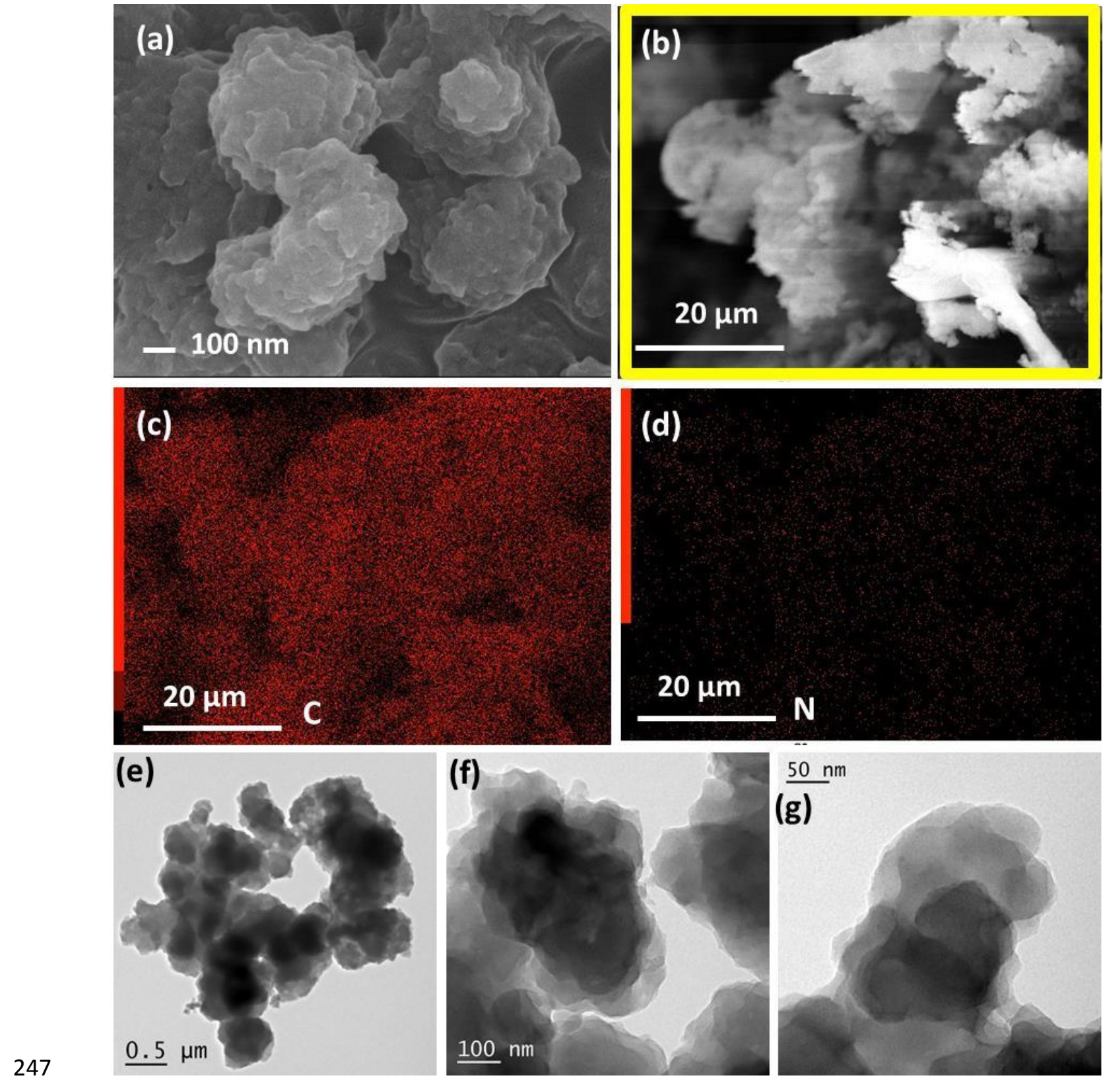

249 Figure S25: (a - d) SEM images and their corresponding elemental mapping of the PT-COF 250 after electrochemical performance. (e-g) TEM images of the COF after GCD cyclic test 251 which indicates retention of the structural morphology compare with the pristine COF. 


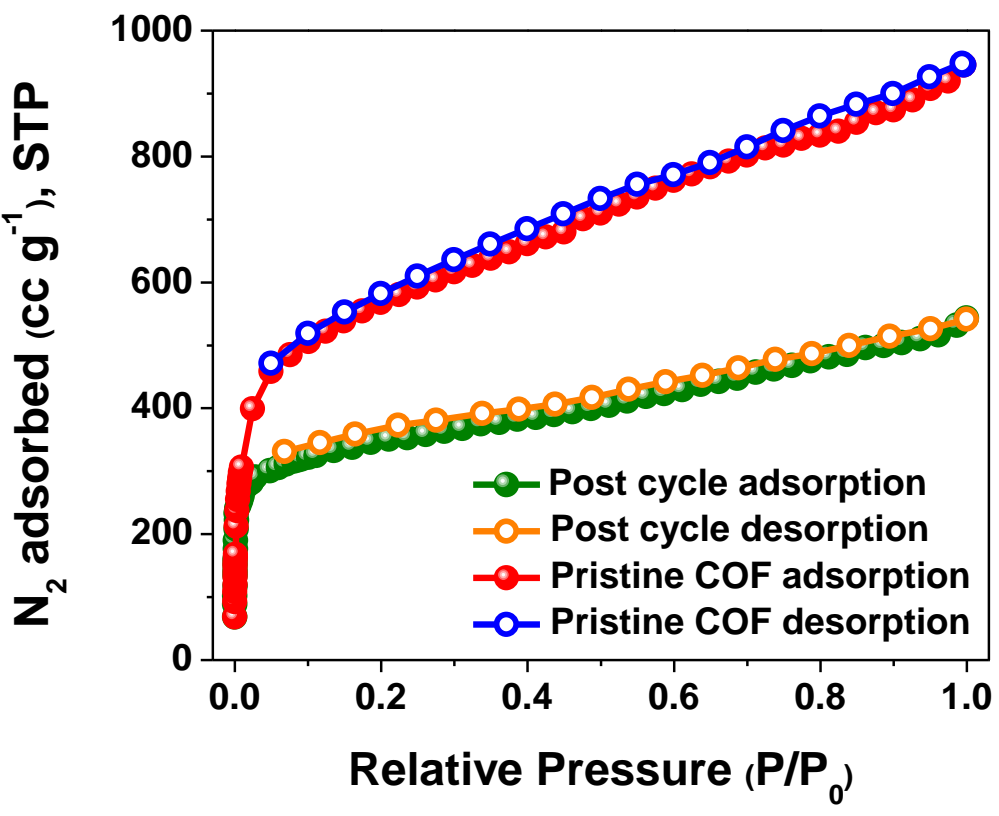

253 Figure S26: $\mathrm{N}_{2}$ adsorption/desorption isotherm of the post cycle sample in comparison with 254 the pristine PT-COF.

S13: References:

1. Dong, J.; Li, X.; Bo Peh, S.; Di Yuan, Y.; Wang, Y.; Ji, D.; Peng, S.; Liu, G.; Ying, S.; Yuan, D.; Jiang, J.; Ramakrishna, S.; Zhao, D. Chem. Mater. 2018, 31, 146-160.

2. Jo, C.; Hwang, I.; Lee, J.; Lee, C. W.; Yoon, S. J. Phys. Chem. C, 2011, 115, 1188011886.

3. Chun, S. E.; Pyun, S. I.; Lee, G. J. Electrochim. Acta, 2006, 51, 6479-6486. 